\title{
Secure Hybrid A/D Beamforming for Hardware-Efficient Large-Scale Multiple-Antenna SWIPT Systems
}

\author{
Yunlong Cai, Fangyu Cui, Qingjiang Shi, Yongpeng Wu, Benoit Champagne, and Lajos Hanzo
}

\begin{abstract}
In this work, we investigate the problem of secure communications in a downlink large-scale multi-antenna assisted simultaneous wireless information and power transfer (SWIPT) system, where a base station (BS) transmits signals to serve a number of information decoding (ID) and energy harvesting (EH) users. Considering that the EH users can potentially eavesdrop the ID users' confidential information, we study the robust joint design of the hybrid analog-digital (A/D) beamforming (BF) matrices and of the artificial redundant signal (ARS) covariance matrix at the $\mathrm{BS}$, where the aim is to maximize the worstcase sum secrecy rate for the ID users under a transmit power constraint, a nonlinear EH constraint and a unit-modulus constraint on the entries of the analog BF matrix. The corresponding optimization problem is very challenging due to the nonlinear and nonconvex objective function and constraints. Using innovative optimization techniques, we first transform the original problem into an equivalent but more tractable form, and then develop a novel joint iterative algorithm based on the penalty-concaveconvex procedure (CCCP) for solving the resultant problem. We show that the proposed penalty-CCCP based algorithm for ARSaided robust joint hybrid BF design converges to a Karush-KuhnTucker solution of the original problem, and also analyze its computational complexity. Our simulation results verify that the resultant robust joint hybrid BF design algorithm relying on ARS significantly outperforms the conventional hybrid BF benchmark
\end{abstract}

The work of Y. Cai was supported in part by the National Natural Science Foundation of China under Grants 61831004 and 61971376, the Zhejiang Provincial Natural Science Foundation for Distinguished Young Scholars under Grant LR19F010002, and the State Key Laboratory of Rail Traffic Control and Safety (Contract No. RCS2020K010), Beijing Jiaotong University. The work of Q. Shi was supported in part by the National Key Research and Development Project under grant 2017YFE0119300, and in part by the National Natural Science Foundation of China under Grants 61671411,61731018 and U1709219. The work of Y. Wu is supported in part by the National Key R\&D Program of China under Grant 2018YFB1801102, JiangXi Key R\&D Program under Grant 20181ACE50028, National Natural Science Foundation of China under Grant 61701301, Young Elite Scientist Sponsorship Program by CAST, and the open research project of State Key Laboratory of Integrated Services Networks (Xidian University) under Grant ISN20-03. L. Hanzo would like to acknowledge the financial support of the Engineering and Physical Sciences Research Council projects EP/N004558/1, EP/P034284/1, EP/P034284/1, EP/P003990/1 (COALESCE), of the Royal Society's Global Challenges Research Fund Grant as well as of the European Research Council's Advanced Fellow Grant QuantCom. (Correspondence authors: Qingjiang Shi.)

Y. Cai is with the Department of ISEE, Zhejiang University, China. He is also with the State Key Laboratory of Rail Traffic Control and Safety, Beijing Jiaotong University, China (e-mail: ylcai521@gmail.com). F. Cui is with ZTE Corporation, Shanghai, China (e-mail: cfy531@zju.edu.cn), Q. Shi is with the School of Software Engineering at Tongji University, Shanghai 201804, China. He is also with the Shenzhen Research Institute of Big Data, Shenzhen 518172, China (e-mail: shiqj@tongji.edu.cn). Y. Wu is with the Department of EE, Shanghai Jiao Tong University, China (e-mail: yongpeng.wu@sjtu.edu.cn). B. Champagne is with the Department of ECE, McGill University, Canada (e-mail: benoit.champagne@mcgill.ca). L. Hanzo is with the Department of ECS, University of Southampton, U.K. (e-mail: lh@ecs.soton.ac.uk). algorithms and efficiently achieves the performance of the fullydigital $B F$ with reduced number of radio frequency chains and energy consumption.

Index Terms-Robust hybrid A/D beamforming, secure communications, large-scale multiple-antenna, energy harvesting, joint optimization.

\section{INTRODUCTION}

\section{A. Literature Review}

Large-scale multiple-antenna techniques have attracted considerable interest for the development of next generation wireless networks [1]-[4], since they can dramatically increase system capacity, hence mitigating the spectrum shortage. $\mathrm{N}$ evertheless, the conventional fully-digital beamforming (BF) structure imposes an excessive fabrication cost and energy consumption owing to using numerous costly radio frequency (RF) chains and analog-digital (A/D) converters. In recent years, a hybrid A/D BF structure has been proposed for circumventing this problem, which allows system developers to rely on a lower number of RF chains than the number of antenna elements [5]-[15].

The main challenges in the design of hybrid $A / D \quad B F$ arise owing to the transmit power constraint with coupling components, imposed by the product of analog and digital BF matrices, and by the unit modulus constraint on the elements of the RF analog BF matrix. By exploiting the structure of large-scale multiple-antenna channels, orthogonal matching pursuit (OMP) and channel matching based hybrid BF (HBF) algorithms have been proposed in [5] and [6], respectively. The authors of [7] and [8] developed joint HBF design algorithms based on manifold optimization, while a design procedure based on the maximization of the system's sum rate was proposed in [9]. The authors of [10] developed a sophisticated joint HBF algorithm based on novel penalty methods, and this algorithm was then further extended to millimeter wave multiple-input multiple-output (MIMO) relay systems in [11]. Additionally, considering the associated hardware limitations, several codebook-based HBF algorithms have been investigated in [12] and [13]. Two-timescale hybrid BF algorithms have been proposed for reducing the overhead in large-scale multiple antenna systems [14], [15], where the long-timescale analog BF matrices are designed based on the channel statistics and the short-timescale digital BF matrices are optimized by using the low-dimensional real-time effective channel state information (CSI) matrices.

Electromagnetic energy harvesting $(\mathrm{EH})$ technologies enabling simultaneous wireless information and power transfer 
(SWIPT) have drawn significant attention as a means to provide cost-effective and perpetual power supply for wireless networks [16]-[20]. The authors of [21] studied a multipleantenna wireless broadcast system consisting of three nodes, where one receiver harvests energy and another receiver decodes information separately from the signals sent by a common transmitter. Two scenarios have been examined, in which the information receiver and energy receiver are separated or co-located. A general receiver operation has been proposed in [22], which splits the received signal with adjustable power ratio for $\mathrm{EH}$ and information decoding (ID) separately. References [23]-[25] investigate a multiuser multiple-input singleoutput (MISO) broadcast SWIPT system configuration, where a multi-antenna base station (BS) sends wireless information and energy signals simultaneously via spatial multiplexing to multiple single-antenna receivers, each implementing either ID or EH. In these works, different optimization problems have been investigated for transmit beamforming design. In [26], a practical nonlinear energy harvesting model and a resource allocation algorithm have been proposed for SWIPT systems.

Considering that the same signal waveform is used for information and energy transmission, security in SWIPT is a major concern, especially within the flourishing context of heterogeneous networks and Internet of things (IoT). Artificial redundant signal (ARS) aided secure BF design algorithms have been developed as a complement to traditional encryption in order to enhance the secrecy performance for the ID users, while supplying energy for the $\mathrm{EH}$ users in multiple-antenna SWIPT systems. Some representative studies on this aspect can be found in [27]-[36]. Furthermore, the security problem in large-scale multiple-antenna assisted SWIPT systems has been investigated in [37]-[42]. Recently, the design of secure HBF for large-scale multiple-antenna systems has also attracted substantial interests [43]-[47]. The authors of [43] proposed a HBF algorithm for minimizing the power leaked to the eavesdroppers while guaranteeing the received signal quality of the legitimate user. In order to maximize the system's secrecy rate, the authors of [44] and [45] studied ARS aided secure HBF algorithms only relying on partial CSI. The design of secure HBF has been investigated in two-way MIMO relay networks in [46]. Moreover, the authors of [47] investigated an energy-efficient HBF algorithm to minimize the total transmit energy and meet the signal-to-noise ratio (SNR) and EH requirements of the ID and $\mathrm{EH}$ users, respectively.

\section{B. Motivation}

Although many studies have been conducted on beamforming for secrecy-preserving SWIPT, most of them are based on the fully-digital beamforming structure which imposes an excessive fabrication cost and energy consumption. Besides, the existing security enhancement techniques conceived for HBF typically design the digital and analog BF matrices separately, which may lead to a significant performance degradation. To the best of our knowledge, the efficient joint design of ARS-aided HBF for secrecy rate maximization in largescale multiple-antenna assisted SWIPT systems has not been previously addressed, and it is well worth studying due to the hardware-efficient hybrid beamforming structure. Against this backdrop, we consider the problem of secure communications in a downlink large-scale multiple-antenna SWIPT system, where a BS transmits signals to serve a number of ID and EH users simultaneously. Considering that the EH users can potentially eavesdrop the ID users' confidential information, we study the joint design of the hybrid A/D BF matrices and of the ARS covariance matrix at the BS, where the aim is to maximize the worst-case sum secrecy rate for the ID users under a transmit power constraint, a nonlinear EH constraint and a unit-modulus constraint on the entries of the analog $\mathrm{BF}$ matrix. The corresponding optimization problem is very challenging due to the nonlinear and nonconvex objective function and constraints. By exploiting the problem structure and using avant-garde techniques, we first transform the original problem into an equivalent yet more tractable form and then develop a novel twin-loop joint iterative algorithm based on the penalty concave-convex procedure (CCCP) to solve the resultant problem. In particular, in the outer loop we adjust the penalty coefficient, while in the inner loop, through insightful approximations and linearization, we optimize the variables in a block coordinate descent fashion, where the subproblems within each block can be solved in parallel. We discuss the convergence and computational complexity of the proposed ARS-aided robust joint HBF design algorithm. Our simulation results show that the proposed algorithm significantly outperforms the existing HBF algorithms and achieves the performance of the fully-digital BF efficiently.

\section{Our Contributions}

The main contributions of this work are summarized as follows:

1) We consider the robust joint design of hybrid $A / D B F$ and ARS covariance matrices to maximize the worst-case secrecy rate, by taking the CSI uncertainties into account. This problem, which has not been previously addressed in the literature, is very challenging due to the nonlinear objective function, the nonconvex transmit power and $\mathrm{EH}$ constraints with coupling terms, and the unit-modulus constraint on the entries of the analog BF matrix. By introducing carefully chosen auxiliary variables, we first convert the challenging constraints into simpler ones, and then transform the objective function into a more tractable form. The resultant problem is equivalent to the original problem but much easier to solve.

2) To solve the transformed problem efficiently, we further introduce a number of auxiliary variables and equality constraints and develop a novel iterative algorithm based on the penalty-CCCP. The proposed algorithm contains two loops, where in the inner loop, through insightful approximations and linearization, the resulting penalized problem is solved in a block coordinate descent fashion to update the optimization variables, while in the outer loop the penalty coefficient is adjusted. Specially, the subproblems within each block of the inner loop can be solved in parallel.

3) We show that the proposed penalty-CCCP-based algorithm for ARS-aided robust joint HBF design converges to a Karush-Kuhn-Tucker (KKT) point of the original 
problem and evaluate its computational complexity. Our simulation results demonstrate the advantages of the newly introduced algorithm over other conventional HBF designs and show that it can achieve the performance of the fully-digital BF with reduced number of RF chains and energy consumption in large-scale multiple-antenna SWIPT systems.

\section{Organization of the Paper}

The paper is structured as follows. Section II briefly describes the system model and formulates the optimization problem of interest in mathematical terms. In Section III, after transforming this problem into a tractable form, the proposed penalty-CCCP based algorithm for robust design of ARS-aided joint HBF in SWIPT systems is derived, and its convergence properties and complexity are discussed. The simulation result$s$ are presented in Section IV and our conclusions are drawn in Section V.

Notations: Scalars, vectors and matrices are respectively denoted by lower case, boldface lower case and boldface upper case letters. I represents an identity matrix and $\mathbf{0}$ denotes an all-zero matrix. For a matrix $\mathbf{A}, \mathbf{A}^{T}, \mathbf{A}^{*}, \mathbf{A}^{H}$ and $\|\mathbf{A}\|$ denote its transpose, conjugate, conjugate transpose and Frobenius norm, respectively. For a square matrix $\mathbf{A}$, $\operatorname{Tr}\{\mathbf{A}\}$ denotes its trace, $\mathbf{A} \succeq \mathbf{0}(\mathbf{A} \preceq \mathbf{0})$ means that $\mathbf{A}$ is positive (negative) semidefinite. For a vector $\mathbf{a},\|\mathbf{a}\|$ represents its Euclidean norm. $E\{$.$\} denotes the statistical expectation.$ $\Re\{\}.(\Im\{\}$.$) denotes the real (imaginary) part of a variable,$ while $|\cdot|$ represents the absolute value of a complex scalar. $\mathbb{C}^{m \times n}\left(\mathbb{R}^{m \times n}\right)$ denotes the space of $m \times n$ complex (real) matrices. The projection function to the nonnegative orthant is denoted by $[a]^{+}=\max (a, 0)$.

\section{SYSTEM MODEL AND PROBLEM FORMULATION}

In this section, we first introduce the model of the hardwareefficient multi-antenna SWIPT system, and then mathematically formulate the optimization problem of interest.

\section{A. System model}

Consider the hardware-efficient multiple-antenna SWIPT system depicted in Fig. 1, which consists of a BS, $J$ ID users and $K \mathrm{EH}$ users which can potentially eavesdrop the confidential information of each ID user. The BS is equipped with $M$ transmit antennas and $N$ RF chains, where $M \geq N$. The ID user and the EH users are equipped with a single antenna.

In order to guarantee the ID users' security while transferring energy to the EH users, a randomly generated ARS vector $\mathbf{f} \in \mathbb{C}^{N \times 1}$ with zero mean and covariance matrix $\mathbf{V}=E\left[\mathrm{ff}^{H}\right]$ is embedded in the baseband signal for transmission. Then, the signal received at the $j$ th ID user, $j \in \mathcal{J} \triangleq\{1, \ldots, J\}$, is given by

$$
y_{D, j}=\mathbf{h}_{j}^{H} \mathbf{U}(\mathbf{f}+\mathbf{W b})+n_{D, j},
$$

where $\mathbf{h}_{j} \in \mathbb{C}^{M \times 1}$ denotes the Hermitian transpose of the flat fading channel vector between the BS and the $j$ th ID user, $\mathbf{U} \in \mathbb{C}^{M \times N}$ denotes the analog BF matrix, $\mathbf{b}=$ $\left[b_{1}, \ldots, b_{J}\right]^{T} \in \mathbb{C}^{J \times 1}$ represents the transmit symbol vector, whose elements are modeled as independent zero-mean circular complex Gaussian random variables with unit variance, i.e., $E\left[\left|b_{j}\right|^{2}\right]=1, \mathbf{W}=\left[\mathbf{w}_{1}, \ldots, \mathbf{w}_{J}\right] \in \mathbb{C}^{N \times J}$ represents the baseband $\mathrm{BF}$ matrix, and $n_{D, j}$ denotes the complex circular Gaussian noise at the $j$ th ID user with zero mean and variance $\sigma_{D, j}^{2}$

The signal received at the $k$ th $\mathrm{EH}$ user, $k \in \mathcal{K} \triangleq$ $\{1, \ldots, K\}$, can be expressed as

$$
y_{E, k}=\mathbf{g}_{k}^{H} \mathbf{U}(\mathbf{f}+\mathbf{W b})+n_{E, k},
$$

where $\mathbf{g}_{k} \in \mathbb{C}^{M \times 1}$ denotes the Hermitian transpose of the channel vector between the BS and the $k$ th $\mathrm{EH}$ user, while $n_{E, k}$ denotes the complex-valued circular Gaussian noise at the $k$ th EH user with zero mean and variance of $\sigma_{E, k}^{2}$.

The transmit power of the BS is given by

$$
E\left[\|\mathbf{U}(\mathbf{f}+\mathbf{W} \mathbf{b})\|^{2}\right]=\|\mathbf{U F}\|^{2}+\|\mathbf{U W}\|^{2},
$$

where based on the positive and Hermitian structure of the ARS covariance matrix, we have introduced a matrix $\mathbf{F} \in$ $\mathbb{C}^{N \times N}$ so that $\mathbf{V}=\mathbf{F F}^{H}$, i.e., $\mathbf{F}$ is the square-root of $\mathbf{V}$.

From (1) and (2), the signal-to-interference plus noise (SINR) at the $j$ th ID user and the SINR at the $k$ th EH user for the $j$ th ID user signal are given as follows, respectively,

$$
\begin{aligned}
& \operatorname{SINR}_{D}^{j} \triangleq \triangleq \frac{\left|\mathbf{h}_{j}^{H} \mathbf{U} \mathbf{w}_{j}\right|^{2}}{\left\|\mathbf{h}_{j}^{H} \mathbf{U F}\right\|^{2}+\sum_{j^{\prime} \neq j}^{J}\left|\mathbf{h}_{j}^{H} \mathbf{U} \mathbf{w}_{j^{\prime}}\right|^{2}+\sigma_{D, j}^{2}}, \\
& \mathbf{S I N R}_{E, k}^{j} \triangleq \frac{\left|\mathbf{g}_{k}^{H} \mathbf{U} \mathbf{w}_{j}\right|^{2}}{\left\|\mathbf{g}_{k}^{H} \mathbf{U F}\right\|^{2}+\sum_{j^{\prime} \neq j}^{J}\left|\mathbf{g}_{k}^{H} \mathbf{U} \mathbf{w}_{j^{\prime}}\right|^{2}+\sigma_{E, k}^{2}} .
\end{aligned}
$$

Besides, considering the nonlinear EH model [48], the total energy that the $k$ th $\mathrm{EH}$ user can harvest is given by

$$
\phi_{k}\left(P_{i n, k}\right)= \begin{cases}\xi P_{i n, k}, & P_{i n, k} \leq P_{s a t, k} \\ \xi P_{s a t, k}, & P_{i n, k}>P_{\text {sat }, k}\end{cases}
$$

where $\xi \in(0,1]$ denotes the energy conversion efficiency of the $k$ th EH user, $P_{s a t, k}$ denotes the saturation threshold of the $k$ th EH receiver, and $P_{i n, k}$ denotes the input RF power, given by

$$
P_{i n, k} \triangleq\left\|\mathbf{g}_{k}^{H} \mathbf{U F}\right\|^{2}+\left\|\mathbf{g}_{k}^{H} \mathbf{U W}\right\|^{2}+\sigma_{E, k}^{2} .
$$

Thus, the maximum harvested energy can be expressed by $\xi P_{\text {sat }, k}$, which is a constant power, when the input power exceeds the threshold $P_{s a t, k}$.

\section{B. Problem formulation}

Since the ID users and the EH users are all authorized network users managed by the BS, the latter can obtain their CSI based on the channel estimates of the users, which are fed back to the BS. However, the CSI errors are inevitable in practice. To characterize this uncertainty, let us consider the commonly used norm-bounded error (NBE) model [49], [50]. Under the NBE model, the true (but unknown) CSI from the BS to the $j$ th ID user, $j \in \mathcal{J}$, and from the BS to the $k$ th $\mathrm{EH}$ user, $k \in \mathcal{K}$, can be expressed as follows:

$$
\mathbf{h}_{j}=\hat{\mathbf{h}}_{j}+\triangle \mathbf{h}_{j}
$$

and

$$
\mathbf{g}_{k}=\hat{\mathbf{g}}_{k}+\triangle \mathbf{g}_{k}
$$




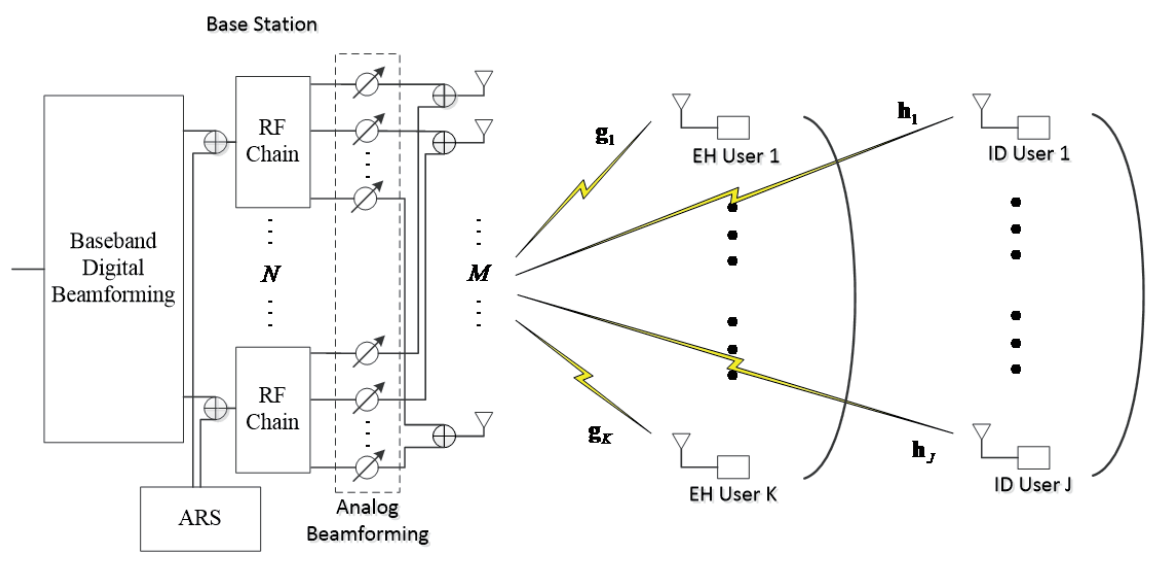

Fig. 1: Hardware-efficient multiple-antenna SWIPT system

where $\hat{\mathbf{h}}_{j}$ and $\hat{\mathbf{g}}_{k}$ denote the imperfect estimates while $\triangle \mathbf{h}_{j}$ and $\triangle \mathbf{g}_{k}$ capture the corresponding uncertainty. In the absence of a priori statistical knowledge about the channel uncertainty, the errors are simply assumed to lie within the following bounded regions

$$
\mathcal{H}_{j}=\left\{\triangle \mathbf{h}_{j}:\left\|\triangle \mathbf{h}_{j}\right\|^{2} \leq \eta_{j}^{2}\right\}
$$

and

$$
\mathcal{R}_{k}=\left\{\triangle \mathbf{g}_{k}:\left\|\triangle \mathbf{g}_{k}\right\|^{2} \leq \varepsilon_{k}^{2}\right\}
$$

where $\eta_{j}$ and $\varepsilon_{k}$ denote the corresponding radii of uncertainty.

The worst-case sum secrecy rate achieved by the ID users is given by (11) [38]. In this contribution we aim for jointly designing the baseband BF matrix $\mathbf{W}$, the ARS shaping matrix $\mathbf{F}$ and the analog BF matrix $\mathbf{U}$ in order to maximize the worstcase sum secrecy rate in (11). Hence, the design problem for the secure hybrid A/D BF can be formulated as (12). The constraint (12b) reflects the limited transmit power budget of the BS, while the constant modulus constraint (12c) is due to the fact that the analog beamformer is implemented using low cost phase shifters. Constraint (12d) guarantees that the energy harvested by the $k$ th $\mathrm{EH}$ user meets a given energy target $\psi_{k}$, where $t_{k}$ denotes the tight lower bound of $P_{i n, k}$ in the presence of CSI errors. We note that the constrained optimization problem (12) is very challenging to solve due to the highly nonconvex objective function and constraints.

\section{Proposed ARS-Aided JoInt Robust HBF Design ALGORITHM}

In this section, we first convert the challenging problem (12) into a more easily tractable yet equivalent form, and then develop a novel penalty-CCCP based iterative algorithm to efficiently obtain its solution.

\section{A. Problem transformation}

The transformation of problem (12) into a simpler yet equivalent form relies on the following lemma whose proof is given in Appendix A.

Lemma 1. Let $\mathbf{G} \in \mathbb{C}^{L \times L}$ be a semi-positive definite Hermitian matrix represented in block form as follows,

$$
\mathbf{G} \triangleq\left[\begin{array}{cc}
x & \overline{\mathbf{x}}^{H} \\
\overline{\mathbf{x}} & \overline{\mathbf{G}}
\end{array}\right] \succeq \mathbf{0}
$$

where $x \in \mathbb{C}, \overline{\mathbf{x}} \in \mathbb{C}^{(L-1) \times 1}$, and $\overline{\mathbf{G}} \in \mathbb{C}^{(L-1) \times(L-1)}$. Then $x \geq 0$ and $\overline{\mathbf{G}} \succeq \mathbf{0}$, and if $x=0$, then $\overline{\mathbf{x}}=\mathbf{0}$.

By defining $\mathbf{X} \triangleq[\mathbf{W}, \mathbf{F}] \in \mathbb{C}^{N \times(J+N)}$, we can represent $\mathbf{W}$ and $\mathbf{F}$ as $\mathbf{W}=\mathbf{X} \boldsymbol{\Omega}_{1}$ and $\mathbf{F}=\mathbf{X} \boldsymbol{\Omega}_{2}$, where $\boldsymbol{\Omega}_{1} \triangleq$ $\left[\mathbf{I}_{J}, \mathbf{0}_{J \times N}\right]^{T}$ and $\boldsymbol{\Omega}_{2} \triangleq\left[\mathbf{0}_{N \times J}, \mathbf{I}_{N}\right]^{T}$ denote the selection matrices. Making use of this notation, we can now state the following theorem, which provides the desired equivalence between (12) and a more tractable problem.

Theorem 1. Problem (12) is equivalent to the following problem in (14), in the sense that the global optimal solution $\mathbf{X}$ and $\mathbf{U}$ for the two problems are identical.

Here $\mathbf{A} \in \mathbb{C}^{M \times M}, \mathbf{B} \in \mathbb{C}^{M \times(N+1)}, \mathbf{C} \in$ $\mathbb{C}^{(N+1) \times(N+1)}, \alpha_{j} \in \mathbb{R}^{+}, \beta_{j} \in \mathbb{R}^{+}$and $\gamma_{j} \in \mathbb{R}^{+}$denote a set of auxiliary variables introduced to simplify the problem solution. $\boldsymbol{\omega}_{j}$ denotes a $J \times 1$ selection vector, where the $j$ th element is one and the remaining elements are zeros.

Proof: Firstly, we show that constraints (12b) and (12c) are equivalent to constraints $(14 \mathrm{~b})-(14 \mathrm{e})$. By defining $\overline{\mathbf{U}} \triangleq$ $\mathbf{U U}^{H}$, constraint (14b) can be rewritten as

$$
\tilde{\mathbf{U}} \triangleq\left[\begin{array}{cc}
\overline{\mathbf{U}}-\mathbf{A} & \mathbf{U X}-\mathbf{B} \\
\mathbf{X}^{H} \mathbf{U}^{H}-\mathbf{B}^{H} & \mathbf{X}^{H} \mathbf{X}-\mathbf{C}
\end{array}\right] \succeq \mathbf{0} .
$$

It is readily seen that $\hat{\mathbf{U}} \triangleq \overline{\mathbf{U}}-\mathbf{A} \succeq \mathbf{0}$. Hence, it follows on the one hand that $\hat{\mathbf{U}}(m, m) \geq 0$, while on the other hand, we have $\hat{\mathbf{U}}(m, m)=\overline{\mathbf{U}}(m, m)-\mathbf{A}(m, m)=\sum_{n=1}^{N}|\mathbf{U}(m, n)|^{2}-$ $\mathbf{A}(m, m)$. Based on constraints (14d) and (14e), we obtain $\hat{\mathbf{U}}(m, m) \leq 0$. Therefore, we must have $\hat{\mathbf{U}}(m, m)=0$.

By considering in turn the upper $M$ diagonal entries of the semi-positive definite matrix $\tilde{\mathbf{U}}$, which are equal to zero as shown above, and repeatedly applying Lemma 1, we obtain $\overline{\mathbf{U}}=\mathbf{A}$ and $\mathbf{B}=\mathbf{U X}$. By substituting $\mathbf{B}=\mathbf{U X}$ into (14c), we obtain the constraint (12b). Moreover, since $\sum_{n=1}^{N}|\mathbf{U}(m, n)|^{2}=\mathbf{A}(m, m) \geq N$, while from $(14 \mathrm{e})$, $|\mathbf{U}(m, n)| \leq 1$, we obtain $|\mathbf{U}(m, n)|=1$, i.e. constraint (12c) must be satisfied.

Secondly, let us turn our attention to the objective function in (12a). By introducing the auxiliary variables $\alpha_{j}, \beta_{j}$ and $\gamma_{j}$, where $\alpha_{j}$ denotes the upper bound of the SINR among the EH users, $\beta_{j} \geq \frac{1}{\alpha_{j}}$, and $\gamma_{j}$ denotes the lower bound of the $j$ th ID user's SINR, we can see that problem (12) can be equivalently 


$$
\begin{aligned}
& \sum_{j=1}^{J}\left[\log \left(1+\min _{\forall \Delta \mathbf{h}_{j} \in \mathcal{H}_{j}} \operatorname{SINR}_{D}^{j}\right)-\log \left(1+\max _{\forall k \in \mathcal{K}} \max _{\forall \Delta \mathbf{g}_{k} \in \mathcal{R}_{k}} \operatorname{SINR}_{E, k}^{j}\right)\right]^{+} . \\
& \max _{\mathbf{W}, \mathbf{F}, \mathbf{U}, t_{k}} \sum_{j=1}^{J} \log \left(1+\min _{\forall \triangle \mathbf{h}_{j} \in \mathcal{H}_{j}} \operatorname{SINR}_{D}^{j}\right)-\sum_{j=1}^{J} \log \left(1+\max _{\forall k \in \mathcal{K}} \max _{\forall \mathbf{g}_{k} \in \mathcal{R}_{k}} \operatorname{SINR}_{E, k}^{j}\right) \\
& \text { s.t. }\|\mathbf{U W}\|^{2}+\|\mathbf{U F}\|^{2} \leq P_{t}, \\
& |\mathbf{U}(m, n)|=1, \forall m, n, \\
& \phi_{k}\left(t_{k}\right) \geq \psi_{k}, P_{i n, k} \geq t_{k}, \forall \triangle \mathbf{g}_{k} \in \mathcal{R}_{k}, \forall k \text {. } \\
& \max _{\substack{\mathbf{X}, \mathbf{U}, \mathbf{A}, \mathbf{B}, \mathbf{C}, \alpha_{j}, \beta_{j}, \gamma_{j}>0, t_{k}}} \sum_{j=1}^{J} \log \left(1+\gamma_{j}\right)-\sum_{j=1}^{J} \log \left(1+\alpha_{j}\right) \\
& \text { s.t. }\left[\begin{array}{c}
\mathbf{U} \\
\mathbf{X}^{H}
\end{array}\right]\left[\begin{array}{ll}
\mathbf{U}^{H} & \mathbf{X}
\end{array}\right] \succeq\left[\begin{array}{cc}
\mathbf{A} & \mathbf{B} \\
\mathbf{B}^{H} & \mathbf{C}
\end{array}\right] \\
& \|\mathbf{B}\|^{2} \leq P_{t} \\
& \mathbf{A}(m, m) \geq N \text {, } \\
& |\mathbf{U}(m, n)| \leq 1, \forall m, n, \\
& \frac{\left|\mathbf{h}_{j}^{H} \mathbf{B} \boldsymbol{\Omega}_{1} \boldsymbol{\omega}_{j}\right|^{2}}{\left\|\mathbf{h}_{j}^{H} \mathbf{B} \boldsymbol{\Omega}_{2}\right\|^{2}+\sum_{j^{\prime} \neq j}^{J}\left|\mathbf{h}_{j}^{H} \mathbf{B} \boldsymbol{\Omega}_{1} \boldsymbol{\omega}_{j^{\prime}}\right|^{2}+\sigma_{D, j}^{2}} \geq \gamma_{j}, \forall \triangle \mathbf{h}_{j} \in \mathcal{H}_{j}, \forall j, \\
& \alpha_{j} \beta_{j} \geq 1, \forall j \text {, } \\
& \frac{\left|\mathbf{g}_{k}^{H} \mathbf{B} \boldsymbol{\Omega}_{1} \boldsymbol{\omega}_{j}\right|^{2}}{\left\|\mathbf{g}_{k}^{H} \mathbf{B} \boldsymbol{\Omega}_{2}\right\|^{2}+\sum_{j^{\prime} \neq j}^{J}\left|\mathbf{g}_{k}^{H} \mathbf{B} \boldsymbol{\Omega}_{1} \boldsymbol{\omega}_{j^{\prime}}\right|^{2}+\sigma_{E, k}^{2}} \leq \frac{1}{\beta_{j}}, \forall \triangle \mathbf{g}_{k} \in \mathcal{R}_{k}, \forall k, \\
& \phi_{k}\left(t_{k}\right) \geq \psi_{k}, \forall k \text {, } \\
& \left\|\mathbf{g}_{k}^{H} \mathbf{B}\right\|^{2}+\sigma_{E, k}^{2} \geq t_{k}, \forall \triangle \mathbf{g}_{k} \in \mathcal{R}_{k}, \forall k \text {. }
\end{aligned}
$$

converted into the following problem:

$$
\begin{aligned}
& \max _{\substack{\mathbf{X}, \mathbf{U}, \mathbf{A}, \mathbf{B}, \mathbf{C}, \alpha_{j}, \beta_{j}, \gamma_{j}>0, t_{k}}} \sum_{j=1}^{J} \log \left(1+\gamma_{j}\right)-\sum_{j=1}^{J} \log \left(1+\alpha_{j}\right) \\
& \text { s.t. }(14 b)-(14 h),(12 d) \text {. }
\end{aligned}
$$

Due to the fact that the constraints (12b) and (12c) are equivalent to constraints (14b)-(14e) and $\mathbf{B}=\mathbf{U X}$, we find that problem (12) can be equivalently transformed into problem (14). This completes the proof.

\section{B. Proposed joint iterative design algorithm}

In this subsection, we develop the proposed penalty-CCCP based iterative algorithm for the efficient joint design of robust secure HBF via the solution of problem (14) and subsequently discuss its properties, including convergence behavior and complexity. The proposed penalty-CCCP based algorithm contains two loops ${ }^{1}$, where the penalty coefficient is adjusted in the outer loop, while in the inner loop the optimization variables are updated in a block coordinate descent fashion. In each block of the inner loop, we aim to decompose the

\footnotetext{
${ }^{1}$ The penalty-CCCP optimization framework is introduced in Appendix B.
}

resulting penalized problem into a number of subproblems, which can be solved easily in parallel. To this end, we introduce auxiliary variables $\mathbf{Y} \in \mathbb{C}^{(M+N+1) \times N}, \mathbf{D} \in$ $\mathbb{C}^{(M+N+1) \times(M+N+1)}, \mathbf{P}_{j} \in \mathbb{C}^{M \times(N+1)}, \tilde{\mathbf{B}}_{k, j} \in \mathbb{C}^{M \times(N+1)}$, $\overline{\mathbf{B}}_{k} \in \mathbb{C}^{M \times(N+1)}, \tilde{\beta}_{k, j}, \bar{t}_{k}$ and $\bar{\phi}_{k}, \forall k, \forall j$, which are subject to the following equality constraints,

$$
\begin{gathered}
\mathbf{Y}=\left[\begin{array}{c}
\mathbf{U} \\
\mathbf{X}^{H}
\end{array}\right], \mathbf{D}=\left[\begin{array}{cc}
\mathbf{A} & \mathbf{B} \\
\mathbf{B}^{H} & \mathbf{C}
\end{array}\right], \\
\mathbf{P}_{j}=\mathbf{B}, \tilde{\mathbf{B}}_{k, j}=\mathbf{B}, \overline{\mathbf{B}}_{k}=\mathbf{B}, \tilde{\beta}_{k, j}=\beta_{j}, \bar{t}_{k}=t_{k}, \\
\bar{\phi}_{k}=\phi_{k}\left(\bar{t}_{k}\right), \forall k, \forall j .
\end{gathered}
$$

Using these variables, we conveniently recast problem (14) as the equivalent form shown in (19), where $\mathcal{S} \triangleq$ $\left\{\mathbf{X}, \mathbf{U}, \mathbf{A}, \mathbf{B}, \mathbf{Y}, \mathbf{P}_{j}, \mathbf{C}, \mathbf{D}, \tilde{\mathbf{B}}_{k, j}, \overline{\mathbf{B}}_{k}, \bar{t}_{k}, t_{k}, \alpha_{j}, \beta_{j}, \tilde{\beta}_{k, j}, \gamma_{j}, \bar{\phi}_{k}\right\}$ denotes the variable set, while $\boldsymbol{\Phi}_{1}=\left[\mathbf{I}_{M}, \mathbf{0}_{M \times(N+1)}\right]$, $\mathbf{\Phi}_{2}=\left[\mathbf{0}_{(N+1) \times M}, \mathbf{I}_{N+1}\right], \quad \mathbf{\Psi}_{1}=\left[\mathbf{I}_{M}, \mathbf{0}_{M \times(N+1)}\right]^{T}$ and $\boldsymbol{\Psi}_{2}=\left[\mathbf{0}_{(N+1) \times M}, \mathbf{I}_{N+1}\right]^{T}$ are selection matrices.

By penalizing the equality constraints (19h)-(19k) into the objective function, we obtain the following penalized problem for (19):

$$
\begin{aligned}
\max _{\mathcal{S}} & \sum_{j=1}^{J} \log \left(1+\gamma_{j}\right)-\sum_{j=1}^{J} \log \left(1+\alpha_{j}\right)-\mathcal{L}_{\rho}(\mathcal{X}) \\
\text { s.t. } & (19 \mathrm{~b})-(19 \mathrm{~g}),
\end{aligned}
$$




$$
\begin{aligned}
& \max _{\mathcal{S}} \sum_{j=1}^{J} \log \left(1+\gamma_{j}\right)-\sum_{j=1}^{J} \log \left(1+\alpha_{j}\right) \\
& \text { s.t. } \quad \mathbf{Y Y}^{H} \succeq \mathbf{D} \text {, } \\
& (14 \mathrm{c})-(14 \mathrm{e}),(14 \mathrm{~g}) \text {, } \\
& \frac{\left|\mathbf{h}_{j}^{H} \mathbf{P}_{j} \boldsymbol{\Omega}_{1} \boldsymbol{\omega}_{j}\right|^{2}}{\left\|\mathbf{h}_{j}^{H} \mathbf{P}_{j} \boldsymbol{\Omega}_{2}\right\|^{2}+\sum_{j^{\prime} \neq j}^{J}\left|\mathbf{h}_{j}^{H} \mathbf{P}_{j} \boldsymbol{\Omega}_{1} \boldsymbol{\omega}_{j^{\prime}}\right|^{2}+\sigma_{D, j}^{2}} \geq \gamma_{j}, \forall \triangle \mathbf{h}_{j} \in \mathcal{H}_{j}, \forall j, \\
& \frac{\left|\mathbf{g}_{k}^{H} \tilde{\mathbf{B}}_{k, j} \boldsymbol{\Omega}_{1} \boldsymbol{\omega}_{j}\right|^{2}}{\left\|\mathbf{g}_{k}^{H} \tilde{\mathbf{B}}_{k, j} \boldsymbol{\Omega}_{2}\right\|^{2}+\sum_{j^{\prime} \neq j}^{J}\left|\mathbf{g}_{k}^{H} \tilde{\mathbf{B}}_{k, j} \boldsymbol{\Omega}_{1} \boldsymbol{\omega}_{j^{\prime}}\right|^{2}+\sigma_{E, k}^{2}} \leq \frac{1}{\tilde{\beta}_{k, j}}, \forall \triangle \mathbf{g}_{k} \in \mathcal{R}_{k}, \forall k, \forall j, \\
& \bar{\phi}_{k} \geq \psi_{k}, \forall k \text {, } \\
& \left\|\mathbf{g}_{k}^{H} \overline{\mathbf{B}}_{k}\right\|^{2}+\sigma_{E, k}^{2} \geq t_{k}, \forall \triangle \mathbf{g}_{k} \in \mathcal{R}_{k}, \forall k \\
& \boldsymbol{\Phi}_{1} \mathbf{Y}=\mathbf{U}, \boldsymbol{\Phi}_{2} \mathbf{Y}=\mathbf{X}^{H} \text {, } \\
& \boldsymbol{\Psi}_{1}^{H} \mathbf{D} \boldsymbol{\Psi}_{2}=\mathbf{B}, \boldsymbol{\Psi}_{2}^{H} \mathbf{D} \boldsymbol{\Psi}_{1}=\mathbf{B}^{H}, \\
& \boldsymbol{\Psi}_{2}^{H} \mathbf{D} \boldsymbol{\Psi}_{2}=\mathbf{C}, \boldsymbol{\Psi}_{1}^{H} \mathbf{D} \boldsymbol{\Psi}_{1}=\mathbf{A} \text {, } \\
& \mathbf{P}_{j}=\mathbf{B}, \tilde{\mathbf{B}}_{k, j}=\mathbf{B}, \overline{\mathbf{B}}_{k}=\mathbf{B}, \tilde{\beta}_{k, j}=\beta_{j}, t_{k}=\bar{t}_{k}, \phi_{k}\left(\bar{t}_{k}\right)=\bar{\phi}_{k}, \forall k, \forall j \text {. } \\
& \mathcal{L}_{\rho}(\mathcal{X}) \triangleq \rho\left(\left\|\boldsymbol{\Phi}_{1} \mathbf{Y}-\mathbf{U}\right\|^{2}+\left\|\boldsymbol{\Phi}_{2} \mathbf{Y}-\mathbf{X}^{H}\right\|^{2}+\left\|\mathbf{\Psi}_{1}^{H} \mathbf{D} \boldsymbol{\Psi}_{1}-\mathbf{A}\right\|^{2}+\left\|\boldsymbol{\Psi}_{1}^{H} \mathbf{D} \boldsymbol{\Psi}_{2}-\mathbf{B}\right\|^{2}\right. \\
& +\left\|\Psi_{2}^{H} \mathbf{D} \Psi_{1}-\mathbf{B}^{H}\right\|^{2}+\left\|\Psi_{2}^{H} \mathbf{D} \Psi_{2}-\mathbf{C}\right\|^{2}+\sum_{j=1}^{J}\left\|\mathbf{P}_{j}-\mathbf{B}\right\|^{2}+\sum_{j=1}^{J} \sum_{k=1}^{K}\left|\beta_{j}-\tilde{\beta}_{k, j}\right|^{2} \\
& \left.+\sum_{j=1}^{J} \sum_{k=1}^{K}\left\|\mathbf{B}-\tilde{\mathbf{B}}_{k, j}\right\|^{2}+\sum_{k=1}^{K}\left\|\mathbf{B}-\overline{\mathbf{B}}_{k}\right\|^{2}+\sum_{k=1}^{K}\left|t_{k}-\bar{t}_{k}\right|^{2}+\sum_{k=1}^{K}\left|\phi_{k}\left(\bar{t}_{k}\right)-\bar{\phi}_{k}\right|^{2}\right) .
\end{aligned}
$$

where $\mathcal{L}_{\rho}(\mathcal{X})$ is expressed as (21) which captures the penalty terms and $\rho$ denotes the penalty coefficient. Moreover, we define $\mathcal{X} \triangleq$ $\left\{\mathbf{X}, \mathbf{U}, \mathbf{A}, \mathbf{B}, \mathbf{Y}, \mathbf{P}_{j}, \mathbf{C}, \mathbf{D}, \tilde{\mathbf{B}}_{k, j}, \overline{\mathbf{B}}_{k}, \beta_{j}, \tilde{\beta}_{k, j}, t_{k}, \bar{t}_{k}, \bar{\phi}_{k}\right\}$. In the proposed penalty-CCCP algorithm, the outer loop adjusts the penalty coefficient $\rho$ and the inner loop solves the penalized problem (20) iteratively based on the CCCP in a block coordinate descent fashion.

In order to handle the nonconvex objective function and the nonconvex constraints shown in (19b), (19d), (19e) and $(19 \mathrm{~g})$, we derive a locally tight lower bound for the objective function along with linearization of the nonconvex constraints. The details are as follows.

1) Lower bound of the objective function: Due to the logarithmic term in its objective function, problem (20) remains difficult to handle. To overcome this difficulty in the local iterative search, we propose to minimize a locally tight upper bound of the objective function instead. Based on the CCCP concept [51], [52], we approximate the function $d_{1}\left(\alpha_{j}\right)=$ $\log \left(1+\alpha_{j}\right)$ in the $i$ th iteration of the proposed algorithm by its first order Taylor expansion around the current value $\alpha_{j}^{i}$, denoted as $\hat{d}_{1}\left(\alpha_{j}^{i}, \alpha_{j}\right)=d_{1}\left(\alpha_{j}^{i}\right)+2 \Re\left\{\nabla d_{1}^{*}\left(\alpha_{j}^{i}\right)\left(\alpha_{j}-\alpha_{j}^{i}\right)\right\}=$ $\log \left(1+\alpha_{j}^{i}\right)+\frac{\left(\alpha_{j}-\alpha_{j}^{i}\right)}{\left(1+\alpha_{j}^{i}\right)}$. Thus, the locally tight lower bound of the objective function in (20) is given by

$$
\sum_{j=1}^{J} \log \left(1+\gamma_{j}\right)-\sum_{j=1}^{J}\left(\log \left(1+\alpha_{j}^{i}\right)+\frac{\left(\alpha_{j}-\alpha_{j}^{i}\right)}{\left(1+\alpha_{j}^{i}\right)}\right)-\mathcal{L}_{\rho}(\mathcal{X}) .
$$

2) Approximation of constraint (19b): Next, let us approximate the nonconvex constraint in (19b). Note that the conventional linearization methods cannot be applied for (19b). However, the following theorem provides a useful alternative.

Theorem 2. A tight lower bound of $\mathbf{Y} \mathbf{Y}^{H}$ is given by $\mathbf{Y}\left(\mathbf{Y}^{i}\right)^{H}+\mathbf{Y}^{i} \mathbf{Y}^{H}-\mathbf{Y}^{i}\left(\mathbf{Y}^{i}\right)^{H}$, where $\mathbf{Y}^{i}$ denotes the value of $\mathbf{Y}$ at the ith iteration.

Proof: Let us consider the following positive semidefinite matrix:

$$
\left(\mathbf{Y}-\mathbf{Y}^{i}\right)\left(\mathbf{Y}-\mathbf{Y}^{i}\right)^{H} \succeq \mathbf{0} .
$$

By expanding the matrix product in (23), we obtain $\mathbf{Y} \mathbf{Y}^{H} \succeq$ $\mathbf{Y} \mathbf{Y}^{i H}+\mathbf{Y}^{i} \mathbf{Y}^{H}-\mathbf{Y}^{i} \mathbf{Y}^{i H}$. This completes the proof.

Therefore, by using Theorem 2 constraint (19b) can be approximated as the following linear matrix inequality (LMI) constraint:

$$
\mathbf{Y} \mathbf{Y}^{i H}+\mathbf{Y}^{i} \mathbf{Y}^{H}-\mathbf{Y}^{i} \mathbf{Y}^{i H} \succeq \mathbf{D}
$$

3) Approximation of constraints (19d), (19e) and (19g) : Let us rewrite the constraint (19e) as the following difference 
of convex (DC) functions:

$$
d_{2}\left(\tilde{\mathbf{B}}_{k, j}\right)-d_{3}\left(\tilde{\mathbf{B}}_{k, j}, \tilde{\beta}_{k, j}\right) \leq 0, \forall k, \forall j,
$$

where $d_{2}\left(\tilde{\mathbf{B}}_{k, j}\right) \triangleq\left|\mathbf{g}_{k}^{H} \tilde{\mathbf{B}}_{k, j} \boldsymbol{\Omega}_{1} \boldsymbol{\omega}_{j}\right|^{2}, \quad d_{3}\left(\tilde{\mathbf{B}}_{k, j}, \tilde{\beta}_{k, j}\right) \triangleq$ $\frac{\left\|\mathbf{g}_{k}^{H} \tilde{\mathbf{B}}_{k, j} \overline{\boldsymbol{\Omega}}_{j}\right\|^{2}+\sigma_{E, k}^{2}}{\tilde{\beta}_{k, j}}$,

$\overline{\boldsymbol{\Omega}}_{j} \triangleq\left[\boldsymbol{\Omega}_{1} \boldsymbol{\omega}_{1}, \ldots, \boldsymbol{\Omega}_{1} \boldsymbol{\omega}_{j-1}, \boldsymbol{\Omega}_{1} \boldsymbol{\omega}_{j+1}, \ldots, \boldsymbol{\Omega}_{1} \boldsymbol{\omega}_{J}, \boldsymbol{\Omega}_{2}\right]$. Using the first order Taylor expansion around $\left(\tilde{\mathbf{B}}_{k, j}^{i}, \tilde{\beta}_{k, j}^{i}\right)$ in the $i$ th iteration, we linearize $d_{3}\left(\tilde{\mathbf{B}}_{k, j}, \tilde{\beta}_{k, j}\right)$ as shown in (26). Then, (19e) can be approximated as the following convex constraint:

$$
d_{2}\left(\tilde{\mathbf{B}}_{k, j}\right)-\hat{d}_{3}\left(\tilde{\mathbf{B}}_{k, j}, \tilde{\beta}_{k, j} ; \tilde{\mathbf{B}}_{k, j}^{i}, \tilde{\beta}_{k, j}^{i}\right) \leq 0, \forall k, \forall j .
$$

By introducing an auxiliary matrix variable $\mathbf{S}_{k, j} \in \mathbb{C}^{M \times M}$ as the upper bound of $\tilde{\mathbf{B}}_{k, j} \boldsymbol{\Omega}_{1} \boldsymbol{\omega}_{j} \boldsymbol{\omega}_{j}^{H} \boldsymbol{\Omega}_{1}^{H} \tilde{\mathbf{B}}_{k, j}^{H}$, i.e.,

$$
\mathbf{S}_{k, j} \succeq \tilde{\mathbf{B}}_{k, j} \boldsymbol{\Omega}_{1} \boldsymbol{\omega}_{j} \boldsymbol{\omega}_{j}^{H} \boldsymbol{\Omega}_{1}^{H} \tilde{\mathbf{B}}_{k, j}^{H}, \forall k, \forall j,
$$

(27) can be equivalently transformed as

$$
\mathbf{g}_{k}^{H} \mathbf{S}_{k, j} \mathbf{g}_{k}-\hat{d}_{3}\left(\tilde{\mathbf{B}}_{k, j}, \tilde{\beta}_{k, j} ; \tilde{\mathbf{B}}_{k, j}^{i}, \tilde{\beta}_{k, j}^{i}\right) \leq 0, \forall k, \forall j .
$$

Note also that (29) must hold for $\forall \triangle \mathbf{g}_{k} \in \mathcal{R}_{k}$, which results in an infinite number of inequalities. By applying the S-procedure [53], (29) can be equivalently converted to the following finite number of LMI constraints shown in (30) and (31), where $\mathbf{W}_{k, j} \triangleq$ $\mathbf{S}_{k, j}-\left(\frac{\tilde{\mathbf{B}}_{k, j} \overline{\mathbf{\Omega}}_{j} \overline{\boldsymbol{\Omega}}_{j}^{H} \tilde{\mathbf{B}}_{k, j}^{i H}+\tilde{\mathbf{B}}_{k, j}^{i} \overline{\boldsymbol{\Omega}}_{j} \overline{\mathbf{\Omega}}_{j}^{H} \tilde{\mathbf{B}}_{k, j}^{H}}{\tilde{\beta}_{k, j}^{i}}-\tilde{\beta}_{k, j} \frac{\tilde{\mathbf{B}}_{k, j}^{i} \overline{\boldsymbol{\Omega}}_{j} \overline{\mathbf{\Omega}}_{j}^{H} \tilde{\mathbf{B}}_{k, j}^{i H}}{\left(\tilde{\beta}_{k, j}^{i}\right)^{2}}\right)$ and $\tau_{k, j}$ is a slack variable.

Furthermore, by using the Schur complement [49], [53], (28) can be equivalently converted to the following LMI constraints

$$
\left[\begin{array}{cc}
\mathbf{S}_{k, j} & \tilde{\mathbf{B}}_{k, j} \boldsymbol{\Omega}_{1} \boldsymbol{\omega}_{j} \\
\boldsymbol{\omega}_{j}^{H} \boldsymbol{\Omega}_{1}^{H} \tilde{\mathbf{B}}_{k, j}^{H} & 1
\end{array}\right] \succeq \mathbf{0}, \forall k, \forall j .
$$

By following the same approach, constraint (19d) can be approximated as the convex one shown in (33) which must hold $\forall \triangle \mathbf{h}_{j} \in \mathcal{H}_{j}$. Based on the S-procedure and Schur complement, we convert (33) into the following finite number of LMI constraints:

$$
\begin{gathered}
{\left[\begin{array}{cc}
-\overline{\mathbf{W}}_{j}+\bar{\tau}_{j} \mathbf{I} & -\overline{\mathbf{W}}_{j} \hat{\mathbf{h}}_{j} \\
-\hat{\mathbf{h}}_{j}^{H} \overline{\mathbf{W}}_{j} & -\sigma_{D, j}^{2}-\hat{\mathbf{h}}_{j}^{H} \overline{\mathbf{W}}_{j} \hat{\mathbf{h}}_{j}-\bar{\tau}_{j} \eta_{j}^{2}
\end{array}\right] \succeq \mathbf{0},} \\
{\left[\begin{array}{cc}
\overline{\mathbf{S}}_{j} & \mathbf{P}_{j} \overline{\mathbf{\Omega}}_{j} \\
\overline{\mathbf{\Omega}}_{j}^{H} \mathbf{P}_{j}^{H} & \mathbf{I}
\end{array}\right] \succeq \mathbf{0}}
\end{gathered}
$$

and

$$
\bar{\tau}_{j} \geq 0,
$$

where $\overline{\mathbf{S}}_{j}$ is an auxiliary variable, $\overline{\mathbf{W}}_{j} \triangleq \overline{\mathbf{S}}_{j}-$ $\left(\frac{\mathbf{P}_{j} \boldsymbol{\Omega}_{1} \boldsymbol{\omega}_{j} \boldsymbol{\omega}_{j}^{H} \boldsymbol{\Omega}_{1}^{H} \mathbf{P}_{j}^{i H}+\mathbf{P}_{j}^{i} \boldsymbol{\Omega}_{1} \boldsymbol{\omega}_{j} \boldsymbol{\omega}_{j}^{H} \boldsymbol{\Omega}_{1}^{H} \mathbf{P}_{j}^{H}}{\gamma^{i}}-\gamma_{j} \frac{\mathbf{P}_{j}^{i} \boldsymbol{\Omega}_{1} \boldsymbol{\omega}_{j} \boldsymbol{\omega}_{j}^{H} \boldsymbol{\Omega}_{1}^{H} \mathbf{P}_{j}^{i H}}{\gamma_{j}^{i 2}}\right)$, and $\bar{\tau}_{j}$ is a slack variable.

Similarly, (19g) can be approximated as the following linear constraints:

$$
\mathbf{g}_{k}^{H} \boldsymbol{\Pi}_{k} \mathbf{g}_{k}+\sigma_{E, k}^{2}-t_{k} \geq 0, \forall \triangle \mathbf{g}_{k} \in \mathcal{R}_{k},
$$

where $\boldsymbol{\Pi}_{k} \triangleq \overline{\mathbf{B}}_{k}^{i} \overline{\mathbf{B}}_{k}^{H}+\overline{\mathbf{B}}_{k} \overline{\mathbf{B}}_{k}^{i H}-\overline{\mathbf{B}}_{k}^{i} \overline{\mathbf{B}}_{k}^{i H}$. Then, by using the S-procedure, (37) can be transformed into the following finite number of LMI constraints,

$$
\left[\begin{array}{cc}
\boldsymbol{\Pi}_{k}+\tilde{\tau}_{k} \mathbf{I} & \boldsymbol{\Pi}_{k} \hat{\mathbf{g}}_{k} \\
\hat{\mathbf{g}}_{k}^{H} \boldsymbol{\Pi}_{k} & -t_{k}+\sigma_{E, k}^{2}+\hat{\mathbf{g}}_{k}^{H} \boldsymbol{\Pi}_{k} \hat{\mathbf{g}}_{k}-\tilde{\tau}_{k} \varepsilon_{k}^{2}
\end{array}\right] \succeq \mathbf{0}, \forall k
$$

$$
\tilde{\tau}_{k} \geq 0, \forall k,
$$

where $\tilde{\tau}_{k}$ denotes a slack variable.

Moreover, we note that constraint $(14 \mathrm{~g})$ can be represented as a second-order cone programming (SOCP) as follows,

$$
\left\|\left[1, \frac{\alpha_{j}-\beta_{j}}{2}\right]\right\| \leq \frac{\alpha_{j}+\beta_{j}}{2}, \forall j .
$$

4) Proposed penalty-CCCP based joint design: By applying (22), (24), (30)-(32), (34)-(36), (38) and (39), we obtain the following approximated convex problem for (20) in the $i$ th iteration of the inner loop:

$$
\begin{aligned}
& \max _{\overline{\mathcal{S}}} \quad \sum_{j=1}^{J} \log \left(1+\gamma_{j}\right)-\sum_{j=1}^{J}\left(\log \left(1+\alpha_{j}^{i}\right)+\frac{\left(\alpha_{j}-\alpha_{j}^{i}\right)}{\left(1+\alpha_{j}^{i}\right)}\right) \\
& \quad-\mathcal{L}_{\rho}(\mathcal{X}) \\
& \text { s.t. } \quad(24),(14 \mathrm{c})-(14 \mathrm{e}),(30)-(32), \\
&(34)-(36),(38)-(40),(19 \mathrm{f}),
\end{aligned}
$$

where $\overline{\mathcal{S}} \triangleq\left\{\mathbf{X}, \mathbf{U}, \mathbf{A}, \mathbf{B}, \mathbf{Y}, \mathbf{P}_{j}, \mathbf{C}, \mathbf{D}, \tilde{\mathbf{B}}_{k, j}, \overline{\mathbf{B}}_{k}, \mathbf{S}_{k, j}, \overline{\mathbf{S}}_{j}, \alpha_{j}\right.$, $\left.\beta_{j}, \tilde{\beta}_{k, j}, \gamma_{j}, \tau_{k, j}, \bar{\tau}_{j}, \tilde{\tau}_{k}, t_{k}, \bar{t}_{k}, \bar{\phi}_{k}\right\}$ denotes the complete set of variables. In each iteration, the optimization variables are divided into the following four blocks, such that for each block the corresponding subproblems can be solved efficiently in parallel.

In Block 1, we optimize $\left\{\tau_{k, j}, \mathbf{S}_{k, j}, \tilde{\mathbf{B}}_{k, j}, \tilde{\beta}_{k, j}\right\}$, $\left\{\tilde{\tau}_{k}, \overline{\mathbf{B}}_{k}, t_{k}\right\}$ and $\left\{\bar{\tau}_{j}, \overline{\mathbf{S}}_{j}, \mathbf{P}_{j}, \gamma_{j}\right\}$ by fixing the other variables. In this block, the corresponding subproblems for $\forall k \in \mathcal{K}$ and $\forall j \in \mathcal{J}$ can be solved in parallel.

In Block 2, we optimize $\mathbf{B},\left\{\alpha_{j}, \beta_{j}\right\}$ and $\left\{\bar{t}_{k}\right\}$ by fixing the other variables. The corresponding subproblems can be solved in closed form.

In Block 3, we optimize $\mathbf{A}, \mathbf{U}, \mathbf{X}$ and $\mathbf{C}$ by fixing the other variables. In this block, we have two constrained subproblems and two unconstrained subproblems, which can be solved in closed form.

In Block 4, we optimize $(\mathbf{X}, \mathbf{D})$ and $\left\{\bar{\phi}_{k}\right\}$ by fixing the other variables.

A detailed derivation for each one of the updating steps of the inner-loop iterative algorithm is provided in Appendix C. The proposed two-loop penalty-CCCP based joint iterative algorithm is summarized in Algorithm 1.

\section{Convergence and complexity}

Based on the proposed approximations and linearization in Subsection III-B and the convergence analysis for penaltyCCCP in [49], we can see that the proposed Algorithm 1 converges to the KKT solution of problem (19). Note that (12) and (19) are equivalent, in the sense that the global optimal solution $(\mathbf{w}, \mathbf{F}, \mathbf{U})$ for the two problems are identical. We therefore conclude that any limit point $\left(\mathbf{w}^{\star}, \mathbf{F}^{\star}, \mathbf{U}^{\star}\right)$ in $\overline{\mathcal{S}}$ of the iterates generated by Algorithm 1 is a KKT point of problem (12).

According to the aforementioned description, the proposed joint design algorithm can be implemented in a parallel manner. We note that the overall computational complexity is dominated by the semidefinite programming (SDP) subproblem with respect to $\left(\bar{\tau}_{j}, \overline{\mathbf{S}}_{j}, \mathbf{P}_{j}, \gamma_{j}\right)$ in Block $\mathbf{1}$ as shown 


$$
\begin{gathered}
\hat{d}_{3}\left(\tilde{\mathbf{B}}_{k, j}, \tilde{\beta}_{k, j} ; \tilde{\mathbf{B}}_{k, j}^{i}, \tilde{\beta}_{k, j}^{i}\right)= \\
{\left[\begin{array}{cc}
-\mathbf{W}_{k, j}+\tau_{k, j} \mathbf{I} & \frac{\operatorname{Tr}\left\{\overline{\mathbf{\Omega}}_{j}^{H} \tilde{\mathbf{B}}_{k, j}^{i H} \mathbf{g}_{k} \mathbf{g}_{k}^{H} \tilde{\mathbf{B}}_{k, j} \overline{\mathbf{\Omega}}_{j}+\overline{\mathbf{\Omega}}_{j}^{H} \tilde{\mathbf{B}}_{k, j}^{H} \mathbf{g}_{k} \mathbf{g}_{k}^{H} \tilde{\mathbf{B}}_{k, j}^{i} \overline{\mathbf{\Omega}}_{j}\right\}}{\tilde{\beta}_{k, j}^{i}}-\tilde{\beta}_{k, j} \frac{\left\|\mathbf{g}_{k}^{H} \tilde{\mathbf{B}}_{k, j}^{i} \overline{\mathbf{\Omega}}_{j}\right\|^{2}+\sigma_{E, k}^{2}}{\left(\hat{\beta}_{k, j}^{i}\right)^{2}}+\frac{2 \sigma_{E, k}^{2}}{\tilde{\beta}_{k, j}^{i}} . \\
\frac{2 \sigma_{E, k}^{2}}{\tilde{\beta}_{k, j}^{i}}-\tilde{\beta}_{k, j} \frac{\sigma_{E, k}^{2}}{\tilde{\beta}_{k, j}^{2}}-\mathbf{W}_{k, j} \hat{\mathbf{g}}_{k} \hat{\mathbf{g}}_{k} \mathbf{W}_{k, j} \hat{\mathbf{g}}_{k}-\tau_{k, j} \varepsilon_{k}^{2}
\end{array}\right] \succeq \mathbf{0}, \forall k, \forall j,} \\
\tau_{k, j} \geq 0, \forall k, \forall j .
\end{gathered}
$$

$$
\left\|\mathbf{h}_{j}^{H} \mathbf{P}_{j} \overline{\boldsymbol{\Omega}}_{j}\right\|^{2}+\sigma_{D, j}^{2}-\left(\frac{\boldsymbol{\omega}_{j}^{H} \boldsymbol{\Omega}_{1}^{H} \mathbf{P}_{j}^{i H} \mathbf{h}_{j} \mathbf{h}_{j}^{H} \mathbf{P}_{j} \boldsymbol{\Omega}_{1} \boldsymbol{\omega}_{j}+\boldsymbol{\omega}_{j}^{H} \boldsymbol{\Omega}_{1}^{H} \mathbf{P}_{j}^{H} \mathbf{h}_{j} \mathbf{h}_{j}^{H} \mathbf{P}_{j}^{i} \boldsymbol{\Omega}_{1} \boldsymbol{\omega}_{j}}{\gamma_{j}^{i}}-\frac{\gamma_{j}\left\|\mathbf{h}_{j}^{H} \mathbf{P}_{j}^{i} \boldsymbol{\Omega}_{1} \boldsymbol{\omega}_{j}\right\|^{2}}{\left(\gamma_{j}^{i}\right)^{2}}\right) \leq 0 .
$$

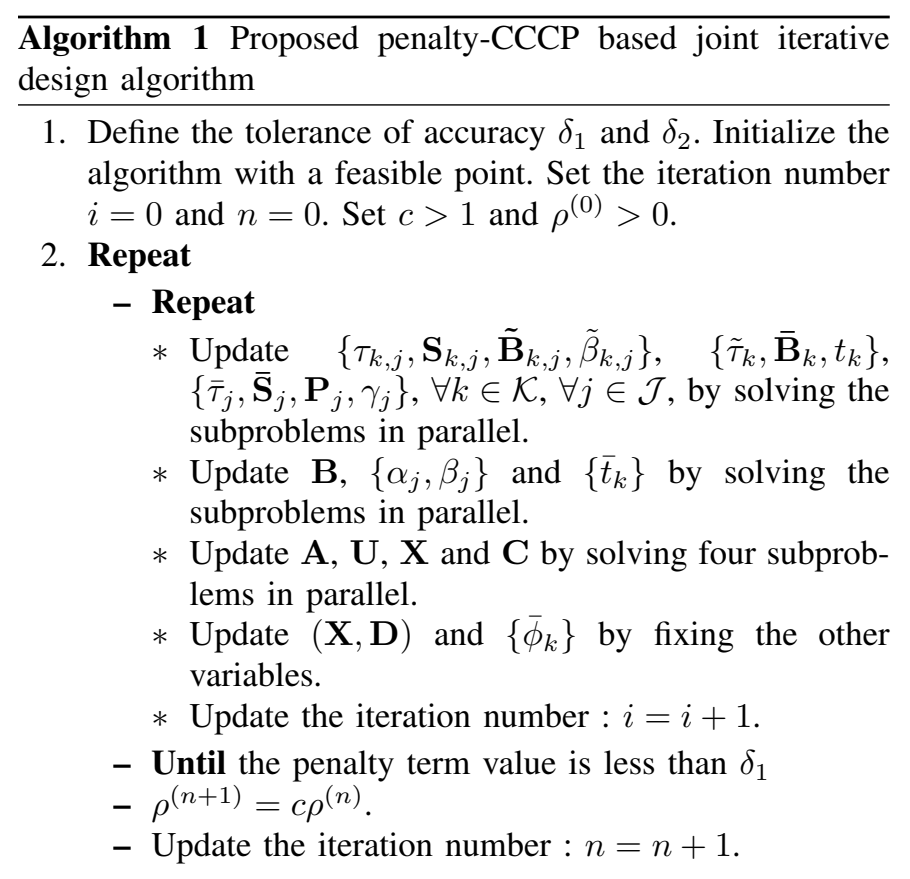

3. Until the difference of successive objective function values is less than $\delta_{2}$ or the maximum number of iterations is reached, i.e., $n>N_{\max }$.

in Appendix C. In this work, we focus on evaluating the order of computational complexity. The complexity of the other subproblems that are solved in closed form or by the method of Lagrange multipliers is much lower than that of the SDP subproblem, hence it can be neglected. Thus, we only concentrate on the main complexity for solving the SDP subproblem which has 1 LMI constraint of size $M+1$ and 1 LMI constraint of size $M+N+J-1$. Hence, the overall complexity of the proposed algorithm is given by

$$
\begin{aligned}
& \mathcal{O}\left(n \sqrt { 2 M + N + J } \left((M+1)^{3}+n(M+1)^{2}\right.\right. \\
& \left.\left.\quad+(M+N+J-1)^{3}+n(M+N+J-1)^{2}+n^{2}\right)\right),
\end{aligned}
$$

where the number of decision variables $n$ is on the order of $\mathcal{O}\left(M^{2}+M(N+J)+2\right)[54]$.

\section{Simulation Results}

In this section, we evaluate the performance of the proposed robust design algorithm for ARS-aided joint HBF. We assume that the BS is equipped with a uniform linear array of $M=32$ transmit antennas and, unless specified otherwise, the transmit power, the number of RF chains, the number of ID users, the number of EH users, the saturation threshold, the energy conversion efficiency, and the EH target are set to $P_{t}=40 \mathrm{dBm}, N=4, J=2, K=2, P_{\text {sat }, k}=-5 \mathrm{dBm}$, $\xi=0.5$, and $\psi=\psi_{k}=-20 \mathrm{dBm}, \forall k \in \mathcal{K}$, respectively. For the large-scale multiple-antenna channel model, we employ the uniform linear antenna array and assume that there are 6 clusters and 8 rays per cluster as in [9], [10]. The large-scale path loss is modeled as (in $\mathrm{dB}$ ) $P L=P L_{0}-10 \alpha \log _{10}\left(d / d_{0}\right)$, where $P L_{0}$ denotes the path loss at reference distance $d_{0}$, $d$ is the link distance in meters, and $\alpha$ denotes the path loss exponent. Here, we set $d_{0}=1$ and $P L_{0}=-30 \mathrm{~dB}$. The distances of the ID and EH users are $d_{I D, j}=500$, $\forall j \in \mathcal{J}$ and $d_{E H, k}=5, \forall k \in \mathcal{K}$, respectively [28]. The path loss exponents regarding the ID and EH users are set as $\alpha_{I D, j}=\alpha_{E H, k}=3.6$ [55]. Moreover, we set the channel bandwidth as $20 \mathrm{MHz}$ and the noise power density as -174 $\mathrm{dBm} / \mathrm{Hz}$. For simplicity, the channel error bounds are assumed to be identical, i.e., $\eta_{j}=\varepsilon_{k}=0.1, \forall j \in \mathcal{J}, \forall k \in \mathcal{K}$. Regarding the implementation of the proposed ARS-aided robust joint HBF design algorithm (Algorithm 1), we set $N_{\max }=30, \rho_{0}=1, c=2, \delta_{1}=10^{-6}$ and $\delta_{2}=10^{-4}$.

Fig. 2 shows the convergence performance of the proposed Algorithm 1. Specifically, Fig. 2 (a) shows the evolution of the sum secrecy rate versus the number of iterations for this algorithm. We can observe that the secrecy rate increases with the number of iterations and converges within 25 iterations, which in this case demonstrates the convergence of the proposed algorithm. Moreover, Fig. 2 (b) indicates the sum of all the penalty terms versus the number of iterations, which supports our claim that the proposed penalty-CCCP based joint design algorithm can tackle the equality constraint effectively.

In the following, the performance of the proposed algorithm is compared with that of other benchmark approaches. Specifically, we consider the following HBF approaches:

- Proposed: The proposed ARS-aided robust joint HBF design algorithm.

- Separate: The analog BF matrix $\mathbf{U}$ is first obtained 


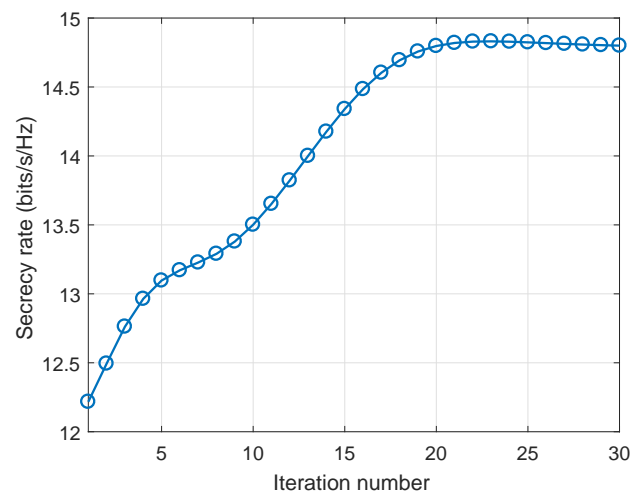

(a)

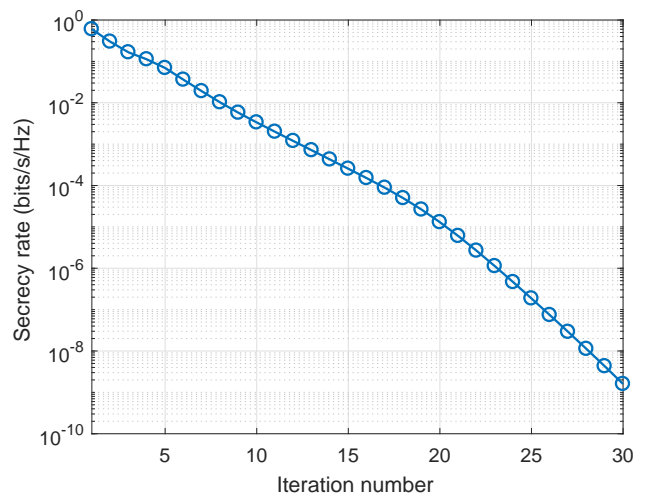

(b)

Fig. 2: The convergence performance of the proposed ARS-aided robust joint HBF design algorithm: (a) Secrecy rate versus iteration number; (b) penalty term versus iteration number.

by using the channel matching approach [6]. The total transmit power is equally allocated to the digital $\mathrm{BF}$ matrix $\mathbf{W}$ and the ARS shaping matrix $\mathbf{F}$, where the former is designed to maximize the ID users' sum rate and the latter is designed in the null space of the ID users' estimated channel matrices and aligned with the EH users estimated channel matrices.

- Without ARS: The analog BF matrix $\mathbf{U}$ and digital BF matrix $\mathbf{W}$ are obtained by the channel matching approach [6] for ID users without the consideration of EH users.

- Fully digital: The ARS-aided robust BF is optimized assuming a fully-digital structure.

- Nonrobust: Simplified version of the proposed ARS-aided joint HBF design algorithm based on estimated CSI only (that is, knowledge of CSI errors is not used).

We first compare the proposed ARS-aided robust joint HBF design algorithm to its nonrobust counterpart. Fig. 3 (a) and (b) demonstrate the secrecy rate and feasible rate versus different values of $\psi$, respectively. In this simulation, the solution produced by a design algorithm is considered feasible for a given channel realization if all the associated constraints are satisfied. The feasible rate represents the proportion (fraction) of simulations experiments where the design algorithm produces a feasible solution in this sense. From the results, the robust algorithm significantly outperforms the nonrobust algorithm for $\psi \leq-15 \mathrm{dBm}$. Although the secrecy rate of the nonrobust algorithm is higher than that of the robust algorithm for $\psi=-10 \mathrm{dBm}$, the proposed robust design algorithm provides $100 \%$ feasible rate which is significantly better than that of its nonrobust counterpart. It shows the effectiveness of the proposed approach against the CSI uncertainties. The improvement in feasible rate comes from the robust operation which takes the CSI error into account.

In Fig. 4, we show the secrecy rate versus the transmit power for the algorithms under comparison. It is seen that the proposed ARS-aided robust joint HBF design algorithm achieves the performance of the fully-digital robust BF algorithm with reduced RF chains and energy consumption. Moreover, the proposed design algorithm significantly outperforms the conventional separate HBF design algorithm and the HBF algorithm without ARS. In particular, for a given secrecy rate of $13 \mathrm{bits} / \mathrm{s} / \mathrm{Hz}$, the proposed algorithm can save almost 3 $\mathrm{dBm}$ in transmit power compared to the conventional separate HBF design method. The secrecy rate of the HBF algorithm without ARS is higher than that of the separate HBF design algorithm for $30 \mathrm{dBm}$ transmit power. That is because the separate HBF design algorithm has to allocate more power to serve the EH users in the case of low transmit power, which degrades the secrecy rate performance. However, the HBF algorithm without ARS does not consider the EH constraints. Fig. 5 shows the secrecy rate performance versus the value of EH target $\psi$. The proposed HBF design algorithm outperforms the separate HBF design algorithm and almost achieves the performance of the fully-digital BF algorithm. The difference between the secrecy rate performance of the proposed ARSaided robust joint HBF design algorithm and that of the fullydigital $\mathrm{BF}$ algorithm increases with the increase of the $\mathrm{EH}$ target value, since more energy is used for wireless power transfer in order to meet the $\mathrm{EH}$ constraint in the hybrid beamforming structure. The number of RF chains can be increased to guarantee the secrecy rate performance for large values of $\mathrm{EH}$ target. Due to the heuristic design without the consideration of EH users, the HBF algorithm without ARS cannot satisfy the EH constraint and its performance does not change with $\psi$.

Subsequently, we investigate the power consumption and energy efficiency performance of the proposed and conventional algorithms. We assume that the levels of power consumption for each low noise amplifier (LNA), RF chain and A/D converter are given by $20 \mathrm{~mW}, 40 \mathrm{~mW}$ and $350 \mathrm{~mW}$, respectively [56]. We also consider that the power consumption of the baseband processing is given by $200 \mathrm{~mW}$. Thus, the power consumption of the fully-digital $\mathrm{BF}$ is given by $M \times(20 \mathrm{~mW}+$ $40 \mathrm{~m} W+350 \mathrm{~mW})+200 \mathrm{~m} W$, while the one of the A/D HBF is given by $M \times 20 m W+N \times 40 m W+N \times 350 m W+200 m W$. By fixing the number of RF chains to $N=8$, the results in Table I show that the HBF structure can significantly reduce the power consumption compared to the fully-digital BF structure, especially as the number of transmit antennas increases. Then, let us define the energy efficiency as $\frac{\tilde{R}}{\tilde{P}}$, 


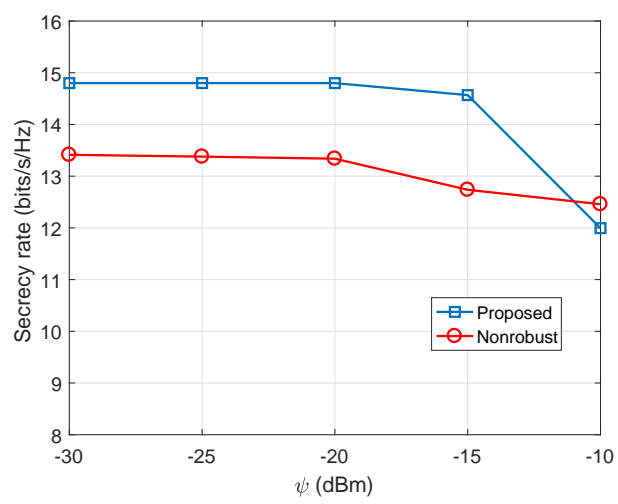

(a)

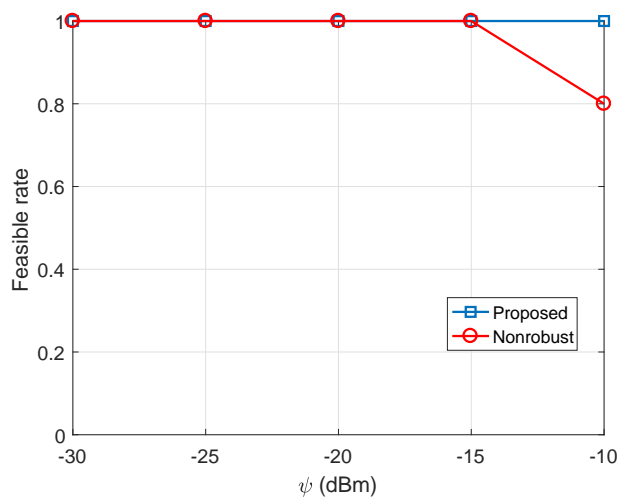

(b)

Fig. 3: Comparison of the proposed ARS-aided robust joint HBF design algorithm and its nonrobust counterpart: (a) Secrecy rate versus $\psi$; (b) feasible rate versus $\psi$.

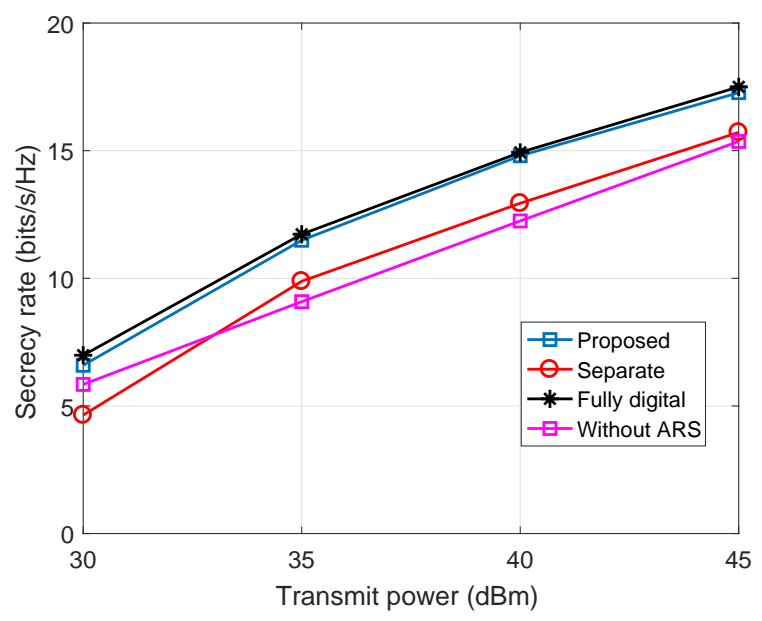

Fig. 4: Comparison of the proposed ARS-aided robust joint HBF design algorithm and the conventional algorithms: Secrecy rate versus transmit power.

where $\tilde{R}$ denotes the secrecy rate of the ID user and $\tilde{P}$ denotes the total power consumption. Fig. 6 shows the energy efficiency for the various algorithms under study versus the number of RF chains. It is seen that the energy efficiency performance of all the HBF approaches decreases with the number of RF chains, and that the proposed ARS-aided robust joint HBF design algorithm outperforms the other compared algorithms and provides the best performance. This is because the proposed HBF design algorithm based on the innovative optimization technique can achieve the same secrecy rate performance as the fully-digital BF with greatly reduced power consumption.

In Fig. 7, we show the secrecy rate performance of the proposed joint hybrid beamforming design algorithm and existing beamforming algorithms versus the number of EH users. From the results, the performance of all the analyzed algorithms degrades with the increase of the number of EH users. The proposed ARS-aided robust joint hybrid beamforming design algorithm outperforms the existing hybrid beamforming al-

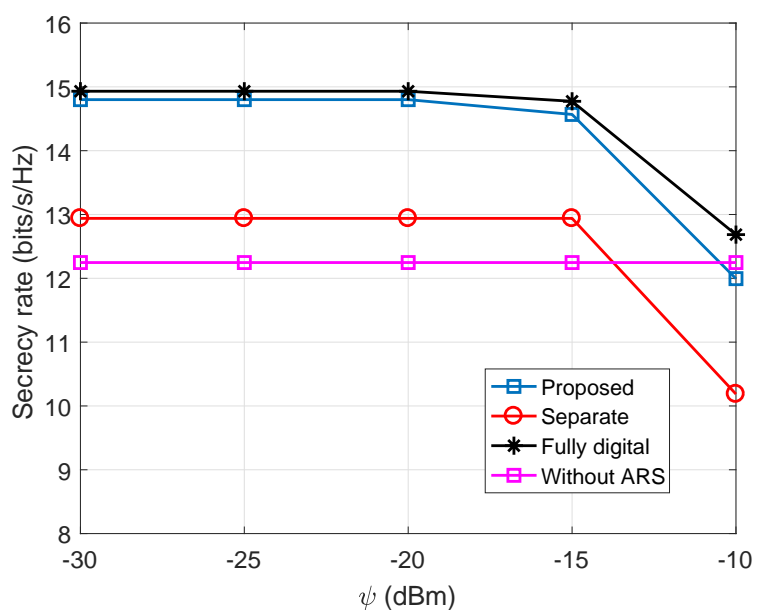

Fig. 5: Comparison of the proposed ARS-aided robust joint HBF design algorithm and the conventional algorithms: Secrecy rate versus the EH target.

gorithms and approaches the performance of the fully-digital beamforming algorithm.

Finally, we investigate the impact of finite resolution phase shifters on the proposed algorithm. Specifically, in Fig. 8, we plot its secrecy rate versus the transmit power for different number $N_{b}$ of phase shifter quantization bits, in the case of $N=4 \mathrm{RF}$ chains and $K=2 \mathrm{EH}$ users. It is seen that the performance of the proposed algorithm improves with the number of quantization bits $N_{b}$ as expected. In particular, the performance with $N_{b}=8$ can approach the performance with infinite resolution phase shifters.

\section{Conclusion}

We have designed ARS-aided robust joint hybrid A/D BF for the secrecy rate maximization of a large-scale multipleantenna assisted SWIPT system. To tackle this challenging multi-dimensional optimization problem, featuring nonlinear objective and nonconvex constraints, we first transformed it into a more tractable, yet equivalent form. We then proposed 
TABLE I: Power consumption for fully-digital and hybrid beamforming structures $(N=8)$

\begin{tabular}{|c|c|c|c|c|}
\hline & $M=32$ & $M=64$ & $M=128$ & $M=256$ \\
\hline Fully Digital & $13.32 W$ & $26.44 W$ & $52.68 W$ & $105.16 \mathrm{~W}$ \\
\hline Hybrid A/D & $3.96 \mathrm{~W}$ & $4.60 \mathrm{~W}$ & $5.88 \mathrm{~W}$ & $8.44 \mathrm{~W}$ \\
\hline
\end{tabular}

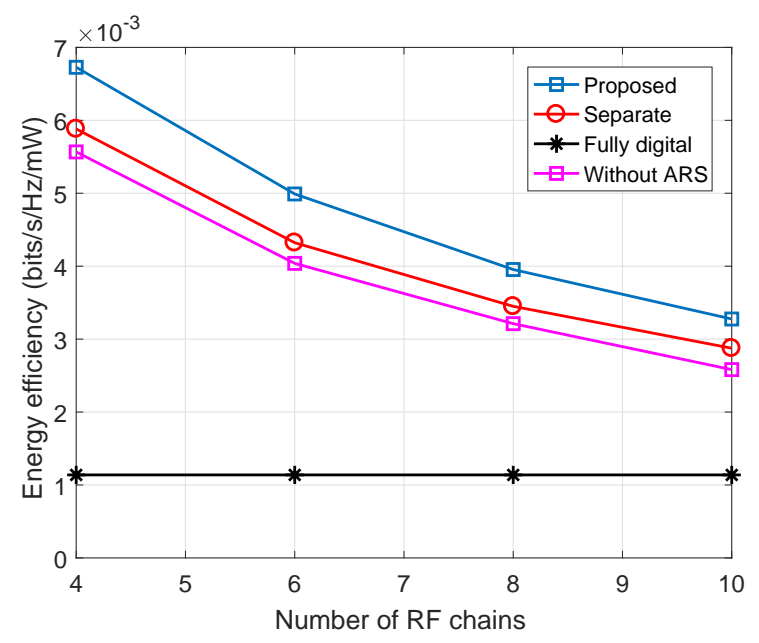

Fig. 6: Comparison of the proposed ARS-aided robust joint HBF design algorithm and the conventional algorithms: Energy efficiency versus number of RF chains.

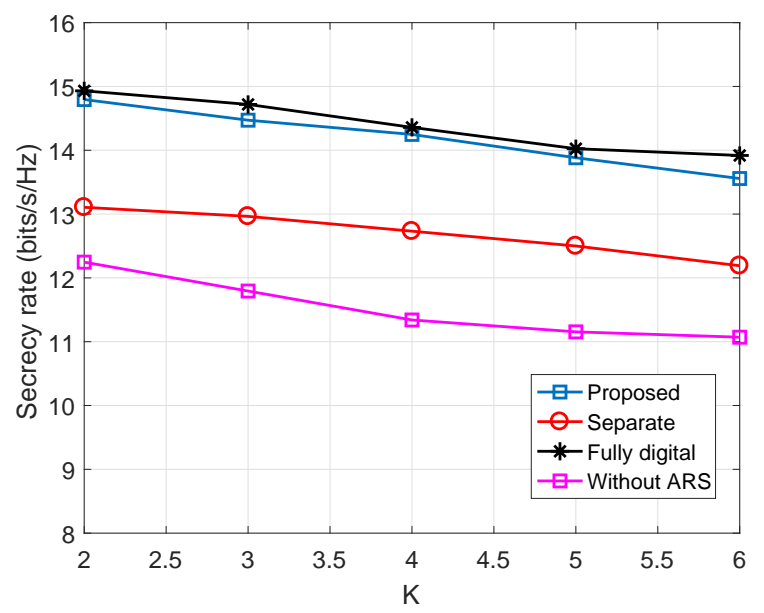

Fig. 7: Comparison of the proposed ARS-aided robust joint HBF design algorithm and the conventional algorithms: Secrecy rate versus number of $\mathrm{EH}$ users.

a penalty-CCCP based twin-loop joint iterative algorithm to solve the resultant problem. Specifically, the penalty coefficient is adjusted in the outer loop, while in the inner loop the optimization variables are updated in a block coordinate descent fashion, where the subproblems within each block can be solved in parallel. We discussed the convergence and computational complexity of the proposed algorithm. Our simulation results have shown that the proposed ARSaided robust joint $\mathrm{HBF}$ design algorithm can outperform the existing HBF benchmark algorithms and efficiently achieves the performance of the fully-digital BF with reduced RF chains and energy consumption.

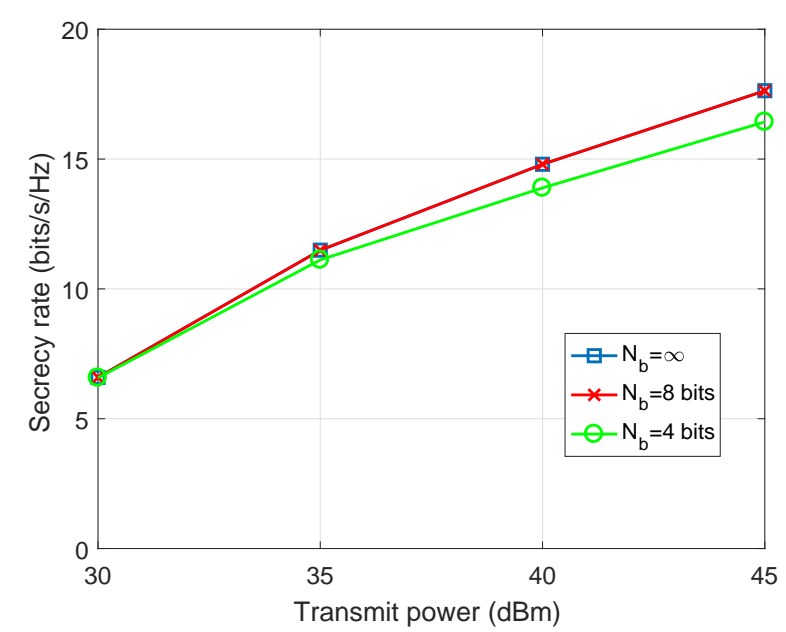

Fig. 8: Secrecy rate of the proposed ARS-aided robust joint $\mathrm{HBF}$ design versus the transmit power with finite resolution phase shifters $(N=4$ and $K=2)$.

\section{APPENDIX A}

PROOF OF LEMMA 1

For any $\mathbf{d} \triangleq\left[\tilde{d}, \overline{\mathbf{d}}^{T}\right]^{T} \in \mathbb{C}^{L \times 1}$, where $\overline{\mathbf{d}} \in \mathbb{C}^{(L-1) \times 1}$, we have

$\mathbf{d}^{H}\left[\begin{array}{cc}x & \overline{\mathbf{x}}^{H} \\ \overline{\mathbf{x}} & \overline{\mathbf{G}}\end{array}\right] \mathbf{d}=x|\tilde{d}|^{2}+\tilde{d} \overline{\mathbf{d}}^{H} \overline{\mathbf{x}}+\tilde{d}^{*} \overline{\mathbf{x}}^{H} \overline{\mathbf{d}}+\overline{\mathbf{d}}^{H} \overline{\mathbf{G}} \overline{\mathbf{d}} \geq 0$.

Hence, by using $\mathbf{d}=\left[0, \overline{\mathbf{d}}^{T}\right]^{T}$, we obtain $\overline{\mathbf{G}} \succeq \mathbf{0}$. Next, we show that when $x=0$ we have $\overline{\mathbf{x}}=\mathbf{0}$ by contradiction.

Assume that when $x=0$ we have $\overline{\mathbf{x}} \neq \mathbf{0}$, then for any $\mathbf{d}$ with $\overline{\mathbf{x}}^{H} \overline{\mathbf{d}} \neq 0$ (43) can be recast as

$$
\tilde{d} \overline{\mathbf{d}}^{H} \overline{\mathbf{x}}+\tilde{d}^{*} \overline{\mathbf{x}}^{H} \overline{\mathbf{d}}+\overline{\mathbf{d}}^{H} \overline{\mathbf{G}} \overline{\mathbf{d}} \geq 0
$$

However, using $\tilde{d}=-\frac{\overline{\mathbf{d}}^{H} \overline{\mathbf{G}} \overline{\mathbf{d}} \overline{\mathbf{x}}^{H} \overline{\mathbf{d}}}{\left|\overline{\mathbf{x}}^{H} \overline{\mathbf{d}}\right|^{2}}$ we have

$$
\tilde{d} \overline{\mathbf{d}}^{H} \overline{\mathbf{x}}+\tilde{d}^{*} \overline{\mathbf{x}}^{H} \overline{\mathbf{d}}+\overline{\mathbf{d}}^{H} \overline{\mathbf{G}} \overline{\mathbf{d}}<0 .
$$

This contradicts (43). Therefore, when $x=0$ we have $\overline{\mathbf{x}}=\mathbf{0}$. Lemma 1 is thus proved.

\section{APPENDIX B \\ PENALTY-CCCP METHOD}

In this appendix, we briefly introduce the proposed penaltyCCCP method in a general framework. Let us consider a general problem:

$$
\begin{array}{r}
(P) \quad \min _{\boldsymbol{x} \in \mathcal{X}} f(\boldsymbol{x}) \\
\text { s.t. } \boldsymbol{h}(\boldsymbol{x})=\mathbf{0}, \\
\boldsymbol{g}(\boldsymbol{x}) \leq \mathbf{0}
\end{array}
$$

where $\mathcal{X} \subseteq \mathbb{R}^{n}$ denotes a closed convex set, $f(\boldsymbol{x})$ is a scalar continuously differentiable function, $\boldsymbol{h}(\boldsymbol{x}) \in \mathbb{R}^{p}$ is a vector of 
TABLE II: Penalty-CCCP method for problem (46)

\begin{tabular}{|l} 
0. \\
1. \\
repeat \\
2. $\quad \boldsymbol{x}^{k+1}=C C C P\left(P_{\varrho_{k}}, \boldsymbol{x}^{k}\right)$ \\
3. $\quad \varrho_{k+1}=c \varrho_{k}$ \\
4. $\quad k=k+1$ \\
5. \\
\end{tabular}

$p$ continuously differentiable functions, and $\boldsymbol{g}(\boldsymbol{x}) \in \mathbb{R}^{q}$ is a vector of differentiable but possibly nonconvex functions.

We can tackle problem $(P)$ by using a penalty method [57], i.e., by solving the penalized problem:

$$
\begin{aligned}
\left(P_{\varrho}\right) \quad & \min _{\boldsymbol{x} \in \mathcal{X}} f_{\rho}(\boldsymbol{x}) \triangleq f(\boldsymbol{x})+\frac{\varrho}{2}\|\boldsymbol{h}(\boldsymbol{x})\|^{2} \\
& \text { s.t. } \boldsymbol{g}(\boldsymbol{x}) \leq \mathbf{0}
\end{aligned}
$$

where $\varrho>0$ is a scalar penalty parameter that prescribes a high cost for the violation of the equality constraints. In particular, when $\varrho \rightarrow \infty$, solving the above problem yields an identical solution to problem $(P)$ [57]. However, it is still difficult to globally solve problem $\left(P_{\varrho}\right)$ when it is a nonconvex problem. Thus, we propose the penalty-CCCP method summarized in Table II to solve problem $(P)$, where in each iteration problem $\left(P_{\varrho}\right)$ is approximately solved using the CCCP method. The resulting solution is denoted simply as $\boldsymbol{x}^{k+1}=C C C P\left(P_{\varrho_{k}}, \boldsymbol{x}^{k}\right)$ in Step 2 of Table II.

Regarding the convergence of penalty-CCCP, this method converges to the KKT solution of the original problem $(P)$. The detailed proof is available from [49].

\section{APPENDIX C}

\section{DERIVATION OF UPDATING STEPS FOR THE INNER-LOOP ALGORITHM}

\section{A. Variables Updating in Block 1}

In this block, problem (41) can be decomposed into a number of independent subproblems, which can be solved in parallel.

1) Subproblems with respect to $\left\{\tau_{k, j}, \mathbf{S}_{k, j}, \tilde{\mathbf{B}}_{k, j}, \tilde{\beta}_{k, j}\right\}$, $\forall k \in \mathcal{K}, \quad \forall j \in \mathcal{J}:$ Each subproblem can be expressed as (48), where $\mathbf{W}_{k, j} \triangleq \mathbf{S}_{k, j}-$ $\left(\frac{\tilde{\mathbf{B}}_{k, j} \overline{\boldsymbol{\Omega}}_{j} \overline{\boldsymbol{\Omega}}_{j}^{H} \tilde{\mathbf{B}}_{k, j}^{i H}+\tilde{\mathbf{B}}_{k, j}^{i} \overline{\boldsymbol{\Omega}}_{j} \overline{\boldsymbol{\Omega}}_{j}^{H} \tilde{\mathbf{B}}_{k, j}^{H}}{\tilde{\beta}_{k, j}^{i}}-\tilde{\beta}_{k, j} \frac{\tilde{\mathbf{B}}_{k, j}^{i} \overline{\boldsymbol{\Omega}}_{j} \overline{\boldsymbol{\Omega}}_{j}^{H} \tilde{\mathbf{B}}_{k, j}^{i H}}{\left(\tilde{\beta}_{k, j}^{i}\right)^{2}}\right)$.

This is a standard SDP problem, which can be solved by using the convex programming toolbox CVX [58].

2) Subproblems with respect to $\left\{\tilde{\tau}_{k}, \overline{\mathbf{B}}_{k}, t_{k}\right\}, \forall k \in \mathcal{K}$ : Each subproblem can be expressed as

$$
\begin{array}{ccc}
\min _{\tilde{\tau}_{k} \geq 0, \overline{\mathbf{B}}_{k}, t_{k}} & \left\|\mathbf{B}-\overline{\mathbf{B}}_{k}\right\|^{2} \\
\text { s.t. } & {\left[\begin{array}{cc}
\boldsymbol{\Pi}_{k}+\tilde{\tau}_{k} \mathbf{I} & \boldsymbol{\Pi}_{k} \hat{\mathbf{g}}_{k} \\
\hat{\mathbf{g}}_{k}^{H} \boldsymbol{\Pi}_{k} & -t_{k}+\sigma_{E, k}^{2}+\hat{\mathbf{g}}_{k}^{H} \boldsymbol{\Pi}_{k} \hat{\mathbf{g}}_{k}-\tilde{\tau}_{k} \varepsilon_{k}^{2}
\end{array}\right] \succeq \mathbf{0}}
\end{array}
$$

where $\boldsymbol{\Pi}_{k} \triangleq \overline{\mathbf{B}}_{k}^{i} \overline{\mathbf{B}}_{k}^{H}+\overline{\mathbf{B}}_{k} \overline{\mathbf{B}}_{k}^{i H}-\overline{\mathbf{B}}_{k}^{i} \overline{\mathbf{B}}_{k}^{i H}$. This SDP problem can be solved by using the toolbox CVX.
3) Subproblems with respect to $\left\{\bar{\tau}_{j}, \overline{\mathbf{S}}_{j}, \mathbf{P}_{j}, \gamma_{j}\right\}$ : This subproblem can be expressed as

$$
\begin{aligned}
& \min _{\bar{\tau}_{j} \geq 0, \overline{\mathbf{S}}_{j}, \mathbf{P}_{j}, \gamma_{j}} \rho\left\|\mathbf{B}-\mathbf{P}_{j}\right\|^{2}-\log \left(1+\gamma_{j}\right) \\
& \text { s.t. } {\left[\begin{array}{cc}
-\overline{\mathbf{W}}_{j}+\bar{\tau}_{j} \mathbf{I} & -\overline{\mathbf{W}}_{j} \hat{\mathbf{h}}_{j} \\
-\hat{\mathbf{h}}_{j}^{H} \overline{\mathbf{W}}_{j} & -\sigma_{D, j}^{2}-\hat{\mathbf{h}}_{j}^{H} \overline{\mathbf{W}}_{j} \hat{\mathbf{h}}_{j}-\bar{\tau}_{j} \eta_{j}^{2}
\end{array}\right] \succeq \mathbf{0}, } \\
& {\left[\begin{array}{cc}
\overline{\mathbf{S}}_{j} & \mathbf{P}_{j} \overline{\boldsymbol{\Omega}}_{j} \\
\overline{\mathbf{\Omega}}_{j}^{H} \mathbf{P}_{j}^{H} & \mathbf{I}
\end{array}\right] \succeq \mathbf{0}, }
\end{aligned}
$$

where $\overline{\mathbf{W}}_{j} \triangleq \overline{\mathbf{S}}_{j}-\left(\frac{\mathbf{P}_{j} \boldsymbol{\Omega}_{1} \boldsymbol{\omega}_{j} \boldsymbol{\omega}_{j}^{H} \boldsymbol{\Omega}_{1}^{H} \mathbf{P}_{j}^{i H}+\mathbf{P}_{j}^{i} \boldsymbol{\Omega}_{1} \boldsymbol{\omega}_{j} \boldsymbol{\omega}_{j}^{H} \boldsymbol{\Omega}_{1}^{H} \mathbf{P}_{j}^{H}}{\gamma^{i}}\right.$ $\left.-\gamma_{j} \frac{\mathbf{P}_{j}^{i} \boldsymbol{\Omega}_{1} \boldsymbol{\omega}_{j} \boldsymbol{\omega}_{j}^{H} \boldsymbol{\Omega}_{1}^{H} \mathbf{P}_{j}^{i H}}{\gamma_{i}^{i 2}}\right)$. This SDP problem can be solved by using the toolbox CVX.

\section{B. Variables Updating in Block 2}

In this block, by fixing the other variables, problem (41) with respect to $\mathbf{B},\left\{\alpha_{j}, \beta_{j}\right\}$ and $\left\{\bar{t}_{k}\right\}, \forall k \in \mathcal{K}, \forall j \in \mathcal{J}$, can be decomposed into several independent subproblems, so that the variables can be optimized in parallel.

1) Subproblems with respect to $\left\{\alpha_{j}, \beta_{j}\right\}$ : Each subproblem is given by

$$
\begin{aligned}
\min _{\alpha_{j}, \beta_{j}} & \frac{\left(\alpha_{j}-\alpha_{j}^{i}\right)}{\left(1+\alpha_{j}^{i}\right)}+\rho \sum_{k=1}^{K}\left|\beta_{j}-\tilde{\beta}_{k, j}\right|^{2} \\
\text { s.t. } & \sqrt{1+\frac{\left(\alpha_{j}-\beta_{j}\right)^{2}}{4}} \leq \frac{\alpha_{j}+\beta_{j}}{2} .
\end{aligned}
$$

By attaching a Lagrange multiplier $\mu$ to the constraint, we obtain the following Lagrange function

$$
\begin{aligned}
\mathcal{L}\left(\alpha_{j}, \beta_{j}, \mu\right) \triangleq & \frac{\left(\alpha_{j}-\alpha_{j}^{i}\right)}{\left(1+\alpha_{j}^{i}\right)}+\rho \sum_{k=1}^{K}\left|\beta_{j}-\tilde{\beta}_{k, j}\right|^{2} \\
& +\mu\left(\sqrt{1+\frac{\left(\alpha_{j}-\beta_{j}\right)^{2}}{4}}-\frac{\alpha_{j}+\beta_{j}}{2}\right) .
\end{aligned}
$$

By examining the first order optimality condition of $\mathcal{L}\left(\alpha_{j}, \beta_{j}, \mu\right)$ with respect to $\alpha_{j}$, we obtain

$$
\alpha_{j}(\mu)=\frac{\left(1+\alpha_{j}^{i}\right) \mu-2}{\sqrt{\left(1+\alpha_{j}^{i}\right) \mu-1}}+\frac{2 \rho \sum_{k=1}^{K} \tilde{\beta}_{k, j}-\frac{1}{1+\alpha_{j}^{i}}+\mu}{2 \rho K} .
$$

Similarly, by examining the first order optimality condition of $\mathcal{L}\left(\alpha_{j}, \beta_{j}, \mu\right)$ with respect to $\beta_{j}$, we have

$$
\beta_{j}(\mu)=\frac{2 \rho \sum_{k=1}^{K} \tilde{\beta}_{k, j}-\frac{1}{1+\alpha_{j}^{i}}+\mu}{2 \rho K} .
$$

The value of the multiplier $\mu$ must be chosen to ensure the complementarity slackness condition of the constraint (51b). In this regard, it is convenient to define the function

$$
\mathcal{C}\left(\alpha_{j}, \beta_{j}\right) \triangleq \sqrt{1+\frac{\left(\alpha_{j}-\beta_{j}\right)^{2}}{4}}-\frac{\alpha_{j}+\beta_{j}}{2} .
$$




$$
\begin{aligned}
\min _{\tau_{k, j} \geq 0, \mathbf{S}_{k, j}, \tilde{\mathbf{B}}_{k, j}, \tilde{\beta}_{k, j}} & \left|\beta_{j}-\tilde{\beta}_{k, j}\right|^{2}+\left\|\mathbf{B}-\tilde{\mathbf{B}}_{k, j}\right\|^{2} \\
\text { s.t. } & {\left[\begin{array}{cc}
-\mathbf{W}_{k, j}+\tau_{k, j} \mathbf{I} & -\mathbf{W}_{k, j} \hat{\mathbf{g}}_{k} \\
-\hat{\mathbf{g}}_{k}^{H} \mathbf{W}_{k, j} & \frac{2 \sigma_{E, k}^{2}}{\tilde{\beta}_{k, j}^{i}}-\tilde{\beta}_{k, j} \frac{\sigma_{E, k}^{2}}{\tilde{\beta}_{k, j}^{i 2}}-\hat{\mathbf{g}}_{k}^{H} \mathbf{W}_{k, j} \hat{\mathbf{g}}_{k}-\tau_{k, j} \varepsilon_{k}^{2}
\end{array}\right] \succeq \mathbf{0}, } \\
& {\left[\begin{array}{cc}
\mathbf{S}_{k, j} & \tilde{\mathbf{B}}_{k, j} \boldsymbol{\Omega}_{1} \boldsymbol{\omega}_{j} \\
\boldsymbol{\omega}_{j}^{H} \boldsymbol{\Omega}_{1}^{H} \tilde{\mathbf{B}}_{k, j}^{H} & 1
\end{array}\right] \succeq \mathbf{0 .} }
\end{aligned}
$$

The optimal $\mu$ should be positive and satisfy $\mathcal{C}\left(\alpha_{j}(\mu), \beta_{j}(\mu)\right)=0$, which is equivalent to

$$
\frac{1}{\sqrt{\left(1+\alpha_{j}^{i}\right) \mu-1}}-\left(\frac{2 \rho \sum_{k=1}^{K} \tilde{\beta}_{k, j}-\frac{1}{1+\alpha_{j}^{i}}+\mu}{2 \rho K}\right)=0 \text {. }
$$

We can obtain the optimal $\mu$ by using a one dimensional search to solve (54). By substituting the resulting value of $\mu$ in (52) and (53), we can obtain the optimal values of $\alpha_{j}$ and $\beta_{j}$.

2) Subproblem with respect to $\mathbf{B}$ : The subproblem is given by

$$
\begin{array}{cl}
\min _{\mathbf{B}} & \left\|\boldsymbol{\Psi}_{1}^{H} \mathbf{D} \boldsymbol{\Psi}_{2}-\mathbf{B}\right\|^{2}+\left\|\boldsymbol{\Psi}_{2}^{H} \mathbf{D} \boldsymbol{\Psi}_{1}-\mathbf{B}^{H}\right\|^{2}+\sum_{j=1}^{J} \| \mathbf{P}_{j}-\mathbf{B} \\
& +\sum_{j=1}^{J} \sum_{k=1}^{K}\left\|\mathbf{B}-\tilde{\mathbf{B}}_{k, j}\right\|^{2}+\sum_{k=1}^{K}\left\|\mathbf{B}-\overline{\mathbf{B}}_{k}\right\|^{2}
\end{array}
$$$$
\text { s.t. }\|\mathbf{B}\|^{2} \leq P_{t} \text {. }
$$

The objective function can be reformulated as $(2 K+3) \| \mathbf{B}-$ $\mathbf{B}_{0} \|^{2}+Z$, where

$$
\begin{aligned}
\mathbf{B}_{0}= & \frac{1}{J K+K+J+2}\left(\boldsymbol{\Psi}_{1}^{H} \mathbf{D} \boldsymbol{\Psi}_{2}+\boldsymbol{\Psi}_{1}^{H} \mathbf{D}^{H} \boldsymbol{\Psi}_{2}+\sum_{j=1}^{J} \mathbf{P}_{j}\right. \\
& \left.+\sum_{j=1}^{J} \sum_{k=1}^{K} \tilde{\mathbf{B}}_{k, j}+\sum_{k=1}^{K} \overline{\mathbf{B}}_{k}\right),
\end{aligned}
$$

and

$$
\begin{aligned}
Z= & \left\|\boldsymbol{\Psi}_{1}^{H} \mathbf{D} \boldsymbol{\Psi}_{2}\right\|^{2}+\left\|\boldsymbol{\Psi}_{1}^{H} \mathbf{D}^{H} \boldsymbol{\Psi}_{2}\right\|^{2}+\sum_{j=1}^{J}\left\|\mathbf{P}_{j}\right\|^{2} \\
& +\sum_{j=1}^{J} \sum_{k=1}^{K}\left\|\tilde{\mathbf{B}}_{k, j}\right\|^{2}+\sum_{k=1}^{K}\left\|\overline{\mathbf{B}}_{k}\right\|^{2}-(J K+K+J+2) \|
\end{aligned}
$$

Therefore, the subproblem (55) is equivalent to

$$
\begin{array}{ll}
\min _{\mathbf{B}} & \left\|\mathbf{B}-\mathbf{B}_{0}\right\|^{2} \\
\text { s.t. } & \|\mathbf{B}\|^{2} \leq P_{t} .
\end{array}
$$

It is readily seen that (58) is equivalent to the projection of $\mathbf{B}_{0}$ onto a hypersphere $\|\mathbf{B}\|^{2} \leq P_{t}$. Thus, the solution is

$$
\mathbf{B}=P_{t} \frac{\mathbf{B}_{0}}{\left\|\mathbf{B}_{0}\right\|+\max \left(0, P_{t}-\left\|\mathbf{B}_{0}\right\|\right)} .
$$

3) Subproblems with respect to $\left\{\bar{t}_{k}\right\}$ : Each subproblem is given by

$$
\min _{\bar{t}_{k}}\left|t_{k}-\bar{t}_{k}\right|^{2}+\left|\phi_{k}\left(\bar{t}_{k}\right)-\bar{\phi}_{k}\right|^{2}
$$

According to the first order optimality condition and the piecewise nature of the function $\phi_{k}\left(\bar{t}_{k}\right)$, the solution for $\bar{t}_{k}$ is given by

$$
\bar{t}_{k}= \begin{cases}t_{k} & \bar{\phi}_{k}>\xi P_{s a t, k} \\ \frac{\xi \bar{\phi}_{k}+t_{k}}{1+\xi^{2}} & \bar{\phi}_{k} \leq \xi P_{s a t, k}\end{cases}
$$

\section{Variables Updating in Block 3}

2 In this block, by fixing the other variables, problem (41) with respect to $\mathbf{A}, \mathbf{U}, \mathbf{X}, \mathbf{C}$ can be decomposed into four independent subproblems, so that the variables in this block can be optimized in parallel.

1) Subproblem 1 with respect to $\mathbf{A}$ : The subproblem is formulated as

$$
\begin{array}{ll}
\min _{\mathbf{A}} & \left\|\boldsymbol{\Psi}_{1}^{H} \mathbf{D} \boldsymbol{\Psi}_{1}-\mathbf{A}\right\|^{2} \\
\text { s.t. } & \mathbf{A}(m, m) \geq N, \forall m .
\end{array}
$$

Based on the first order optimality condition, we obtain $\mathbf{A}_{0}=$ $\boldsymbol{\Psi}_{1}^{H} \mathbf{D} \boldsymbol{\Psi}_{1}$. For the diagonal elements in $\mathbf{A}$, we set

$$
\mathbf{A}(m, m)=\max \left(\mathbf{A}_{0}(m, m), N\right), \forall m .
$$

For the elements that are not on the diagonal, we set

$$
\mathbf{A}(m, n)=\mathbf{A}_{0}(m, n), \forall m \neq n .
$$

2) Subproblem 2 with respect to $\mathbf{C}$ : The subproblem is given by

$$
\min _{\mathbf{C}}\left\|\Psi_{2}^{H} \mathbf{D} \Psi_{2}-\mathbf{C}\right\|^{2}
$$

By applying the first order optimality condition, the optimal C can be obtained as

$$
\mathbf{C}=\Psi_{2}^{H} \mathbf{D} \Psi_{2}
$$

3) Subproblem 3 with respect to $\mathbf{U}$ : The subproblem is given as

$$
\begin{array}{ll}
\min _{\mathbf{U}} & \left\|\mathbf{U}-\mathbf{U}_{0}\right\|^{2} \\
\text { s.t. } & |\mathbf{U}(m, n)| \leq 1, \forall m, n,
\end{array}
$$

where we define $\mathbf{U}_{0} \triangleq \boldsymbol{\Phi}_{1} \mathbf{Y}$. The subproblem (62) can be further divided into $M N$ parallel subproblems. For each element $\mathbf{U}(m, n)$, we have the following subproblem:

$$
\begin{aligned}
\min _{\mathbf{U}(m, n)} & \left\|\mathbf{U}(m, n)-\mathbf{U}_{0}(m, n)\right\|^{2} \\
\text { s.t. } & |\mathbf{U}(m, n)| \leq 1 .
\end{aligned}
$$


Similar to subproblem (55), the subproblem (63) is equivalent to a projection. Therefore, we can obtain

$$
\mathbf{U}(m, n)=\frac{\mathbf{U}_{0}(m, n)}{\left|\mathbf{U}_{0}(m, n)\right|+\max \left(0,1-\left|\mathbf{U}_{0}(m, n)\right|\right)}, \forall m, n .
$$

4) Subproblem 4 with respect to $\mathbf{X}$ : The subproblem is given as

$$
\min _{\mathbf{X}}\left\|\boldsymbol{\Phi}_{2} \mathbf{Y}-\mathbf{X}^{H}\right\|^{2}
$$

By applying the first order optimality condition, the optimal $\mathrm{X}$ can be obtained as

$$
\mathbf{X}=\mathbf{Y}^{H} \boldsymbol{\Phi}_{2}^{H} .
$$

\section{Variables Updating in Block 4}

In this block, problem (41) can be decomposed into the following subproblems, which can be solved in parallel.

1) Subproblem with respect to $(\mathbf{Y}, \mathbf{D})$ : This subproblem is given by

$$
\begin{array}{ll}
\min _{\mathbf{Y}, \mathbf{D}} & \left\|\boldsymbol{\Phi}_{1} \mathbf{Y}-\mathbf{U}\right\|^{2}+\left\|\mathbf{\Phi}_{2} \mathbf{Y}-\mathbf{X}^{H}\right\|^{2}+\left\|\mathbf{\Psi}_{1}^{H} \mathbf{D} \Psi_{1}-\mathbf{A}\right\|^{2} \\
& +\left\|\mathbf{\Psi}_{1}^{H} \mathbf{D} \Psi_{2}-\mathbf{B}\right\|^{2}+\left\|\mathbf{\Psi}_{2}^{H} \mathbf{D} \boldsymbol{\Psi}_{1}-\mathbf{B}^{H}\right\|^{2} \\
& +\left\|\Psi_{2}^{H} \mathbf{D} \Psi_{2}-\mathbf{C}\right\|^{2} \\
\text { s.t. } \quad \mathbf{Y} \mathbf{Y}^{i H}+\mathbf{Y}^{i} \mathbf{Y}^{H}-\mathbf{Y}^{i} \mathbf{Y}^{i H} \succeq \mathbf{D} .
\end{array}
$$

This is a standard SDP problem, which can be solved by using the toolbox CVX.

2) Subproblems with respect to $\left\{\bar{\phi}_{k}\right\}$ : Each subproblem is given by

$$
\begin{gathered}
\min _{\bar{\phi}_{k}}\left|\phi_{k}\left(\bar{t}_{k}\right)-\bar{\phi}_{k}\right|^{2} \\
\text { s.t. } \quad \psi_{k} \leq \bar{\phi}_{k} \leq \xi P_{\text {sat }, k} .
\end{gathered}
$$

By applying the first order optimality condition, the optimal $\bar{\phi}_{k}$ can be obtained as

$$
\bar{\phi}_{k}=\min \left(\xi P_{s a t, k}, \max \left(\psi_{k}, \phi_{k}\left(\bar{t}_{k}\right)\right)\right) \text {. }
$$

\section{REFERENCES}

[1] Z. Pi and F. Khan, "An introduction to millimeter-wave mobile broadband systems," IEEE Commun. Mag., vol. 49, no. 6, pp. 101-107, Jun. 2011.

[2] S. Rangan, T. S. Rappaport, and E. Erkip, "Millimeter-wave cellular wireless networks: Potentials and challenges," Proc. IEEE, vol. 102, no. 3, pp. 366-385, Mar. 2014.

[3] M. R. Akdeniz, Y. Liu, M. K. Samimi, S. Sun, S. Rangan, T. S. Rappaport, and E. Erkip, "Millimeter wave channel modeling and cellular capacity evaluation," IEEE J. Sel. Areas Commun., vol. 32, no. 6, pp. 1164-1179, Jun. 2014.

[4] I. Hemadeh, K. Satyanarayana, M. El-Hajjar, and L. Hanzo, "Millimeterwave communications: Physical channel models, design considerations, antenna constructions and link-budget," IEEE Commun. Surv. Tut., vol. 20, no. 2, pp. 870-913, second quarter, 2018.

[5] O. El Ayach, R. W. Heath, Jr., S. Abu-Surra, S. Rajagopal, and Z. Pi, "Low complexity precoding for large millimeter wave MIMO systems," in Proc. ICC, Jun. 2012, pp. 3724-3729.

[6] J. Zhang, M. Haardt, I. Soloveychik, and A. Wiesel, "A channel matching based hybrid analog-digital strategy for massive multi-user MIMO downlink systems," in Proc. IEEE Sensor Array Multichannel Signal Process. Workshop, Jul. 2016, pp. 1-5.

[7] X. Yu, J.-C. Shen, J. Zhang, and K. B. Letaief, "Alternating minimization algorithms for hybrid precoding in millimeter wave MIMO systems," IEEE J. Sel. Topics Signal Process., vol. 10, no. 3, pp. 485-500, Apr. 2016.
[8] X. Zhai, Y. Cai, Q. Shi, M. Zhao, G. Y. Li, and B. Champagne, "Joint transceiver design with antenna selection for large-scale MU-MIMO millimeter-wave systems," IEEE J. Sel. Areas Commun., vol. 35, no. 9, pp. 2085-2096, Sep. 2017.

[9] F. Sohrabi and W. Yu, "Hybrid digital and analog beamforming design for large-scale antenna arrays," IEEE J. Sel. Topics in Signal Process., vol. 10, no. 3, pp. 501-513, Apr. 2016.

[10] Q. Shi and M. Hong, "Spectral efficiency optimization for mmWave multiuser MIMO systems," IEEE J. Sel. Toptics Signal Process., vol. 12 no. 3, pp. 455-468, Jun. 2018.

[11] Y. Cai, Y. Xu, Q. Shi, B. Champagne, and L. Hanzo, "Robust joint hybrid transceiver design for millimeter wave full-duplex MIMO relay systems," IEEE Trans. Wireless Commun., vol. 18, no. 2, pp. 1199-1215, Feb. 2019.

[12] S. He, J. Wang, Y. Huang, B. Ottersten, and W. Hong, "Codebookbased hybrid precoding for millimeter wave multiuser systems," IEEE Trans. Signal Process., vol. 65, no. 20, pp. 5289-5304, Oct. 15, 2017.

[13] C. Lin, G. Y. Li, and L. Wang, "Subarray-based coordinated beamforming training for mmWave and sub-THz communications," IEEE J. Sel. Areas Commun., vol. 35, no. 9, pp. 2115-2126, Jun. 2017.

[14] A. Liu, V. K. N. Lau, and M. Zhao, "Stochastic successive convex optimization for two-timescale hybrid precoding in massive MIMO," IEEE J. Sel. Toptics Signal Process., vol. 12, no. 3, pp. 432-444, Jun. 2018.

[15] X. Chen, A. Liu, Y. Cai, V. K. N. Lau, and M. Zhao, "Randomized two-timescale hybrid precoding for downlink multicell massive MIMO systems," IEEE Trans. Signal Process., vol. 67, no. 16, pp. 4152-4167, Aug. 15, 2019.

[16] L. R. Varshney, "Transporting information and energy simultaneously," in Proc. IEEE Int. Symp. Inf. Theory (ISIT), Jul. 2008, pp. 1612-1616.

[17] P. Grover and A. Sahai, "Shannon meets Tesla: Wireless information and power transfer,' in Proc. IEEE Int. Symp. Inf. Theory (ISIT), Jun. 2010, pp. 2363-2367.

[18] P. Popovski and O. Simeone, "Two-way communication with energy exchange," in Proc. IEEE Inf. Theory Workshop, Sep. 2012, pp. 592596

[19] I. Krikidis, S. Timotheou, S. Nikolaou, G. Zheng, D. W. K. Ng, and R. Schober, "Simultaneous wireless information and power transfer in modern communication systems," IEEE Commun. Mag., vol. 52, no. 11, pp. 104-110, Nov. 2014.

[20] S. Bi, C. K. Ho, and R. Zhang, "Wireless powered communication: Opportunities and challenges," IEEE Commun. Mag., vol. 53, no. 4, pp. 117-125, Apr. 2015.

[21] R. Zhang and C. K. Ho, "MIMO broadcasting for simultaneous wireless information and power transfer," IEEE Trans. Wireless Commun., vol. 12, no. 5, pp. 1989-2001, May 2013.

[22] X. Zhou, R. Zhang, and C. K. Ho, "Wireless information and power transfer: Architecture design and rate-energy tradeoff," IEEE Trans. Commun., vol. 61, no. 11, pp. 4754-4767, Nov. 2013.

[23] J. Xu, L. Liu, and R. Zhang, "Multiuser MISO beamforming for simultaneous wireless information and power transfer," IEEE Trans. Signal Process., vol. 62, no. 18, pp. 4798-4810, Sep. 15, 2014.

[24] Q. Shi, L. Liu, W. Xu, and R. Zhang, "Joint transmit beamforming and receive power splitting for MISO SWIPT systems," IEEE Trans. Wireless Commun., vol. 13, no. 6, pp. 3269-3280, Jun. 2014.

[25] L. Liu, R. Zhang, and K.-C. Chua, "Multi-antenna wireless powered communication with energy beamforming," IEEE Trans. Commun., vol. 62, no. 12, pp. 4349-4361, Dec. 2014.

[26] E. Boshkovska, D. W. K. Ng, N. Zlatanov, and R. Schober, "Practical non-linear energy harvesting model and resource allocation for SWIPT systems," IEEE Commun. Lett., vol. 19, no. 12, pp. 2082-2085, Dec. 2015.

[27] L. Liu, R. Zhang, and K.-C. Chua, "Secrecy wireless information and power transfer with MISO beamforming," IEEE Trans. Signal Process., vol. 62, no. 7, pp. 1850-1863, Apr. 1, 2014.

[28] M. Alageli, A. Ikhlef, and J. Chambers, "Optimization for maximizing sum secrecy rate in MU-MISO SWIPT systems," IEEE Trans. Veh. Technol., vol. 67, no. 1, pp. 537-553, Jan. 2018.

[29] R. Liu and W. Trappe, Securing Wireless Communications at the Physical Layer. Springer, Norwell, MA, USA, 2009.

[30] Y. Wu, R. Schober, D. W. K. Ng, C. Xiao, and G. Caire, "Secure massive MIMO transmission with an active eavesdropper," IEEE Trans. Inf. Theory, vol. 62, no. 7, pp. 3880-3900, Jul. 2016.

[31] A. Khisti and G. Wornell, "Secure transmission with multiple antennasI: The MISOME wiretap channel," IEEE Trans. Inf. Theory, vol. 56, no. 7, pp. 3088-3104, Jul. 2010 
[32] A. Khisti and G. Wornell, "Secure transmission with multiple antennasII: The MIMOME wiretap channel," IEEE Trans. Inf. Theory, vol. 56, no. 11, pp. 5515-5532, Nov. 2010.

[33] Q. Shi, W. Xu, J. Wu, E. Song, and Y. Wang, "Secure beamforming for MIMO broadcasting with wireless information and power transfer," IEEE Trans. Wireless Commun., vol. 14, no. 5, pp. 2841-2853, May 2015.

[34] Z. Zong, H. Feng, F. R. Yu, N. Zhao, T. Yang, and B. Hu, "Optimal transceiver design for SWIPT in K-user MIMO interference channels," IEEE Trans. Wireless Commun., vol. 15, no. 1, pp. 430-445, Jan. 2016.

[35] Y. Lu, K. Xiong, P. Fan, Z. Zhong, and K. B. Letaief, "Robust transmit beamforming with artificial redundant signals for secure SWIPT system under non-linear EH model," IEEE Trans. Wireless Commun., vol. 17 no. 4, pp. 2218-2232, Apr. 2018.

[36] Y. Lu, K. Xiong, P. Fan, Z. Zhong, and K. B. Letaief, "Coordinated beamforming with artificial noise for secure SWIPT under non-linear EH model: centralized and distributed designs," IEEE J. Sel. Areas Commun., vol. 36, no. 7, pp. 1544-1563, Jul. 2018.

[37] Y. Wu, A. Khisti, C. Xiao, G. Caire, K.-K, Wong, and X. Gao, "A survey of physical layer security techniques for $5 \mathrm{G}$ wireless networks and challenges ahead," IEEE J. Sel. Areas Commun., vol. 36, no. 4, pp. 679-695, Apr. 2018.

[38] A. Mukherjee, S. A. A. Fakoorian, J. Huang, and A. L. Swindlehurst, "Principles of physical layer security in multiuser wireless networks: A survey," IEEE Commun. Surv. Tut., vol. 16, no. 3, pp. 1550-1573, third quarter, 2014

[39] Y. Wu, C. Xiao, Z. Ding, X. Gao, and J. Shi, "Linear precoding for finite-alphabet signaling over MIMOME wiretap channels," IEEE Trans. Veh. Technol., vol. 61, no. 6, pp. 2599-2612, Jul. 2012.

[40] Y. Wu, J.-B. Wang, J. Wang, R. Schober, and C. Xiao, "Secure transmission with large numbers of antennas and finite alphabet inputs," IEEE Trans. Commun., vol. 65, no. 8, pp. 3614-3628, Aug. 2017.

[41] X. Chen, D. W. K. Ng, W. Gerstacker, and H-H. Chen, "A survey of multiple-antenna techniques for physical layer security," IEEE Commun. Surv. Tut., vol. 19, no. 2, pp. 1027-1053, Jun.2017.

[42] X. Chen, D. W. K. Ng, and H-H. Chen, "Secrecy wireless information and power transfer: Challenges and opportunities," IEEE Wireless Commun. Mag., vol. 23, no. 2, pp. 54-61, Apr. 2016.

[43] Y. Huang, J. Zhang, and M. Xiao, "Constant envelope hybrid precoding for directional millimeter-wave communications," IEEE J. Sel. Areas Commun., vol. 36, no. 4, pp. 845-859, Apr. 2018.

[44] Y. R. Ramadan, H. Minn, and A. S. Ibrahim, "Hybrid analog-digital precoding design for secrecy mmWave MISO-OFDM systems," IEEE Trans. Commun., vol. 65, no. 11, pp. 5009-5026, Nov. 2017.

[45] Y. R. Ramadan and H. Minn, "Artificial noise aided hybrid precoding design for secure mmWave MISO systems with partial channel knowledge,' IEEE Signal Process. Lett., vol. 24, no. 11, pp. 1729-1733, Nov. 2017.

[46] S. Gong, C. Xing, Z. Fei, and S. Ma, "Millimeter-wave secrecy beamforming designs for two-way amplify-and-forward MIMO relaying networks," IEEE Trans. Veh. Technol., vol. 66, no. 3, pp. 2059-2071, Mar. 2017.

[47] A. Li and C. Masouros, "Energy-efficient SWIPT: From fully digital to hybrid analog-digital beamforming," IEEE Trans. Veh. Technol., vol. 67, no. 4, pp. 3390-3405, Apr. 2018.

[48] Y. Dong, M. J. Hossain and J. Cheng, "Performance of wireless powered amplify and forward relaying over nakagami- $m$ fading channels with nonlinear energy harvester,' IEEE Commun. Lett., vol. 20, no. 4, pp. 672-675, Apr. 2016.

[49] Y. Cai, Q. Shi, B. Champagne, and G. Y. Li, "Joint transceiver design for secure downlink communications over an amplify-and-forward MIMO relay," IEEE Trans. Commun., vol. 65, no. 9, pp. 3691-3704, Sep. 2017.

[50] P. Ubaidulla and A. Chockalingam, "Relay precoder optimization in MIMO-relay networks with imperfect CSI," IEEE Trans. Signal Process., vol. 59, no. 11, pp. 5473-5484, Nov. 2011.

[51] A. L. Yuille and A. Rangarajan, "The concave-convex procedure," Neural Computation, vol. 15, no. 4, pp. 915-936, Apr. 2003.

[52] G. R. Lanckriet and B. K. Sriperumbudur, "On the convergence of the concave-convex procedure," in Advances in Neural Info. Processing Systems, pp. 1759-1767, 2009.

[53] S. Boyd and L. Vandenberghe, Convex Optimization. Cambridge, U.K.: Cambridge Univ. Press, 2004.

[54] K.-Y. Wang, A. Man-Cho So, T.-H. Chang, W.-K. Ma, and C.-Y. Chi, "Outage constrained robust transmit optimization for multiuser MISO downlinks: Tractable approximations by conic optimization," IEEE Trans. Signal Process., vol. 62, no. 21, pp. 5690-5705, Nov. 2014.

[55] Q. Wu and R. Zhang, "Intelligent reflecting surface enhanced wireless network via joint active and passive beamforming," IEEE Trans. Wireless Commun., vol. 18, no. 11, pp. 5394-5409, Nov. 2019.
[56] T. S. Rappaport, R.W. Heath, Jr., J. N. Murdock, and R. C. Daniels, Millimeter Wave Wireless Communications, Pearson, 2014

[57] D. Bertsekas, Nonlinear Programming, 2nd ed. Belmont, MA, USA: Athena Scientific, 1999.

[58] CVX Research, Inc. CVX: Matlab software for disciplined convex programming, version 2.0 beta. [Online]. Available: http://cvxr.com/cvx 